\title{
Computational models of sprouting angiogenesis and cell migration: towards multiscale mechanochemical models of angiogenesis
}

\author{
T. A. M. Heck ${ }^{a}$, M. M. Vaeyens ${ }^{a} 1$ and H. Van Oosterwyck ${ }^{a, b} 2$ \\ ${ }^{a}$ Biomechanics Section, Department of Mechanical Engineering, KU Leuven, Leuven, Belgium \\ ${ }^{b}$ Prometheus, Division of Skeletal Tissue Engineering, KU Leuven, O\&N 1, Leuven, Belgium
}

\begin{abstract}
Angiogenesis, the formation of new bloods vessels from the existing vasculature, is a process that is essential during development and regeneration of tissues, and that plays a major role in diseases like cancer. Computational models have been designed to obtain a better understanding of the mechanisms behind angiogenesis. In this paper we review computational models of sprouting angiogenesis. These models can be subdivided into three categories: models that mainly focus on tip cell migration, models that make a distinction between the role of tip cells and stalk cells, and models that consider cell shape dynamics. Many models combine discrete modeling of individual cells with continuous modeling of the extracellular matrix (ECM) and diffusing solutes, in this way resulting in a hybrid model. We discuss their merits in unraveling the role of certain factors for vascular network formation, such as the role of (chemotactic, haptotactic, contact) guidance cues in the dynamics and morphology of vascular network formation, and the role of cell-cell interactions that govern tip cell selection and phenotypic changes in general. At the same time, we identify a need for the inclusion of cell mechanical principles in models of angiogenesis, in particular for the description of cell migration, cell-matrix and cell-cell interaction, as the generation of cellular forces is key to cell migration. To further underline this we review models of single cell migration that incorporate such principles, which could be the starting point for formulating novel models of angiogenesis that respect the fundamental laws of classical mechanics at the cell level. As the generation of cellular forces is strongly mediated by pro-angiogenic signals, such models must couple cell mechanical principles to molecular signaling into multiscale mechanochemical models of angiogenesis. Finally, a tight coupling between models and experiments will be required to facilitate model improvements and the generation of novel insights on the regulation of angiogenesis.
\end{abstract}

Key words: angiogenesis, cell migration, cell mechanics, molecular signaling, computational

\footnotetext{
${ }^{1}$ Joint first author

${ }^{2}$ Corresponding author. E-mail: hans.vanoosterwyck@kuleuven.be
} 
models

AMS subject classification: $12 \mathrm{~A} 34,56 \mathrm{~B} 78$

\section{Introduction}

Angiogenesis is the formation of new blood vessels from the pre-existing vascular network. This natural phenomenon occurs during development of healthy tissue and wound healing, but is also typically a hallmark of cancer. When the distance between (tumor) cells and the most proximate blood vessel exceeds the oxygen diffusion distance, the cells become hypoxic and release angiogenic factors such as vascular endothelial growth factor (VEGF) to promote angiogenesis. Cells need this vascularization not only for oxygen transport, but also for nutrient supply and for the disposal of metabolic waste. Sprouting angiogenesis is one of the modes by which normal tissues and tumors make new blood vessels (apart from other methods, such as vasculogenesis or intussusception, see e.g. [21] for a description of these and other modes) characterized by sprouts composed of endothelial cells (ECs) growing towards an angiogenic stimulus. It is regulated by numerous signaling cascades triggered by growth factors such as VEGF, and it can be further modulated by interactions between the ECM and the ECs, as well as between cells.

After a brief description of the basic steps and (molecular) mechanisms of sprouting angiogenesis, we will review computational models of sprouting angiogenesis and their merits in increasing our understanding of its governing mechanisms. The emphasis will be on hybrid models, which combine different mathematical and computational representations and implementations for coupling various aspects of angiogenesis. Often, it refers to the combination of continuum-type (such as partial differential equations) and discrete representations (such as agents) of interacting entities. At the same time, these hybrid models are characterized by some multiscale aspect, where the different representations are often used to describe phenomena at different (length) scales, such as phenomena that are taking place (or that are averaged over) the extracellular scale, cell scale or molecular scale.

Besides EC proliferation and elongation, sprout growth relies on migration of the ECs that constitute a sprout. Cell migration depends on the cell's ability to generate and apply forces to the ECM, which is directly related to the dynamics of cell adhesion and the cell's cytoskeleton [137]. Despite the prominent role of cell-matrix mechanical interactions, we will show that only a few computational models of sprouting angiogenesis explicitly deal with cell mechanical forces. The inclusion of mechanical laws into mathematical and computational models of multicellular organization is not new and has been pioneered by Oster, Murray and co-workers several decades ago, who established continuum-type mechanochemical models to study morphogenesis (see e.g. $[88,92,94]$ for a more in dept discussion). By including cell contractility-driven advective transport into (taxis-)reaction-diffusion equations of cell and matrix density and complementing these equations with a continuum mechanical framework (balance of momentum, constitutive and kinematic equations), they were able to show that cell-matrix mechanical interactions are impor- 
tant determinants of morphogenesis, as taking place during e.g. vasculogenesis [81]. While a more in depth treatment of these continuum-type models falls outside the scope of this review (which is focusing on hybrid models), we can still be inspired by Murrays motivation why models of cell and tissue organization should care about classical mechanics (see also [88]):

- Mechanics acts as additional feedback to increase the stability (robustness) of a biological process.

- Cell and tissue organization must obey classical mechanics. As these laws are very strict and unambiguous, they will put additional constraints on a biological system, therefore limiting the solution space.

- The inclusion of mechanical variables leads to additional opportunities for model validation, as simulated and measured mechanical behaviors must agree.

While only few studies exist that have imposed mechanical laws in their models of sprouting angiogenesis, it is quite the opposite for computational models of single cell migration. Because of the importance of cell migration for angiogenesis and in order to facilitate cross-fertilization between both modeling applications, we will also review computational models of single cell migration that deal with cell mechanical principles.

From this combined review of models of angiogenesis and single cell migration, we will formulate a number of recommendations and challenges for future work, which have to do with combining cell mechanical principles with molecular signaling downstream of a pro-angiogenic signal, effectively resulting into mechanochemical (mechanobiological) models of angiogenesis.

\section{A brief description of sprouting angiogenesis}

During formation of novel vessel branches, several phases of sprouting angiogenesis can be distinguished, as reviewed elaborately by other authors $[19,20,39,43]$ and illustrated in Figure 1. In response to hypoxia, VEGF will be secreted by hypoxic cells and will diffuse through the surrounding ECM, thereby creating a gradient (which will among others depend on the affinity of the ligand to the ECM). When reaching a blood vessel, VEGF can induce phenotypic changes in ECs that line the blood vessel, turning a quiescent EC into a highly motile and polarized tip cell that initiates an angiogenic sprout. A tip cell hardly proliferates, but migrates forward, navigates with its filopodia, secretes ECM degrading enzymes and invades the surrounding tissue. Neighboring cells are inhibited to become a tip-cell by Notch signaling, and become stalk cells which proliferate and migrate, thereby contributing to sprout elongation. In the growing sprout, migration of ECs is observed for both tip and stalk cells and a dynamic interchange between these cell types has been demonstrated in vitro [64], and in vivo [3,64]. Proliferation of the stalk cells allows sprout elongation. When new sprouts come into contact with other sprouting vessels, the tip cells can fuse in a process called anastomosis. The formation of a vascular lumen allows blood flow, and thus tissue oxygenation, leading to a decrease in VEGF expression levels. The novel vessels then 


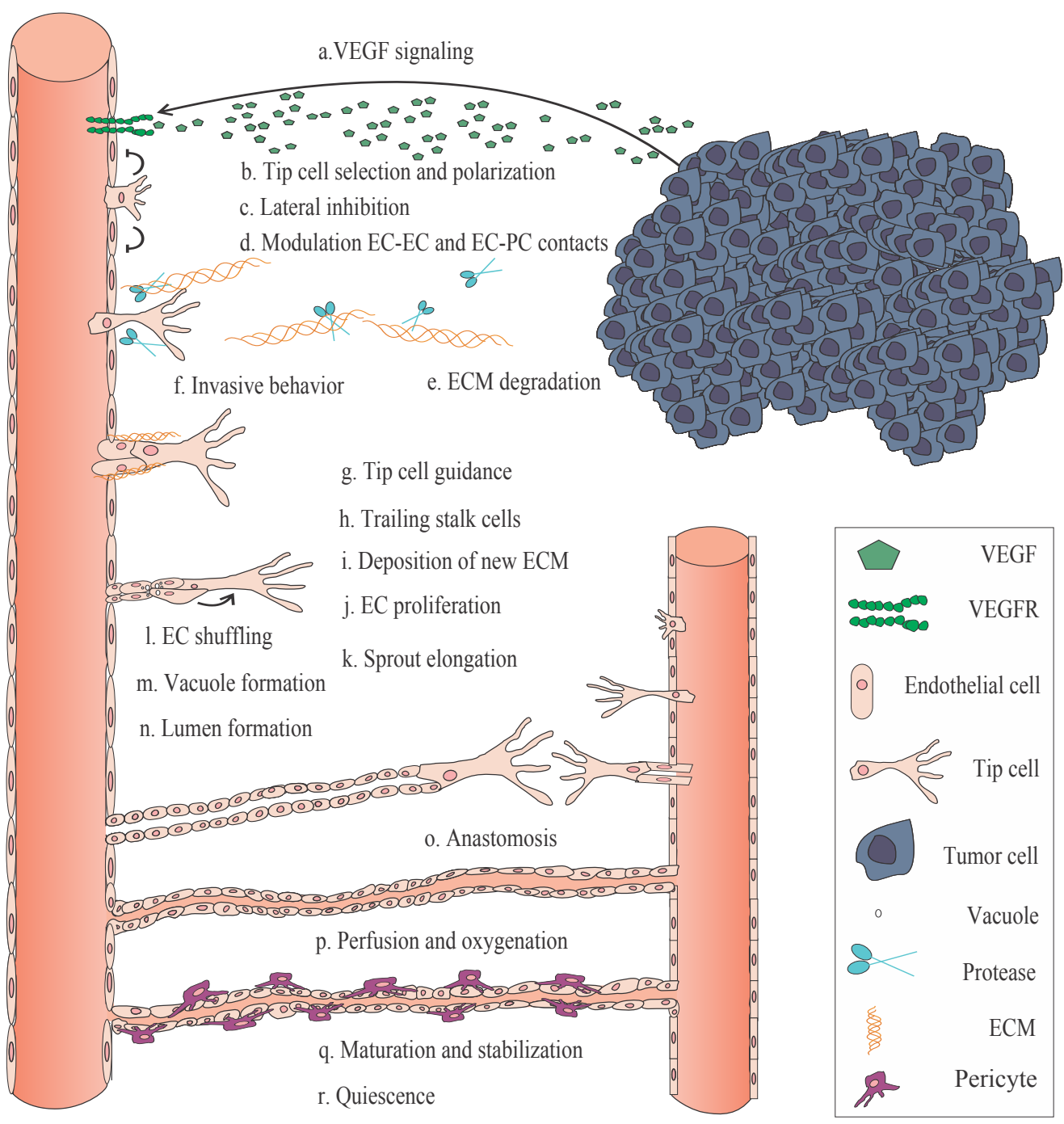

Figure 1: Different phases of sprouting angiogenesis: a) In response to hypoxia, tumor cells will secrete proangiogenic signals such as VEGF, which diffuse through the surrounding ECM. b) When reaching a blood vessel, VEGF binds to receptors on ECs aligning the blood vessel, hereby inducing phenotype changes turning a quiescent EC into a highly motile and polarized tip cell that initiates an angiogenic sprout. c) Neighboring cells are inhibited to become a tip cell by Notch signaling. d) The junctions between ECs and between ECs and pericytes are modulated. e) The ECM is degraded by proteolytic enzymes secreted by the ECs. f) The tip cell shows invasive behavior. g) The tip cell hardly proliferates, but migrates forward, navigates with its filopodia and guides the growing sprout into the surrounding tissue. h) The leading tip cell is followed by stalk cells trailing behind, which proliferate and migrate, thereby contributing to sprout elongation. i) Deposition of new basement membrane. j) The ECs proliferate and k) the stalk elongates. 1) Tip cells can be overtaken by new cells in a process called EC shuffling. m) Vacuoles are formed and $n$ ) merge to form a vascular lumen, allowing blood flow, and thus tissue oxygenation, leading to a decrease in VEGF expression levels. o) Tip cells encountering other tips cells fuse in a process called anastomosis. p) The vessel gets perfused and oxygenated. q) The vessel matures and is stabilized by the recruitment of pericytes and deposition of ECM. r) Finally the ECs of the new vessel recover a quiescent endothelial phalanx phenotype. 
mature and are stabilized by the recruitment of pericytes and deposition of ECM. In a final phase the ECs adopt a quiescent endothelial phalanx phenotype [19].

The most prominent growth factor in the angiogenic sprouting process is VEGF, which triggers an extensive network of signaling cascades displaying complex crosstalk with other pathways involved in cell migration, proliferation and survival. A selection of key molecular players and interactions is illustrated in Figure 2.

Apart from chemical signals, mechanical properties of the ECM are important regulators of key cell processes during angiogenesis as well, such as lamellipodium and filopodium formation [59], alignment and elongation of vascular cells [53] and the apoptotic switch [59]. Other ECM properties such as pore size, matrix density and number and distribution of ligands also contribute to successful migration and sprouting of cells. An excellent review on effects of matrix mechanical properties on angiogenesis has been published by Shiu et al. [115].

\section{Computational models of angiogenesis}

In order to elucidate the governing mechanisms behind the formation of a vascular network, computational models of angiogenic sprouting have been designed. Some of the earlier models have described ECs and the vascular network as continuous density fields, hereby neglecting the structural details of the formed network of sprouts (See e.g. [2] for a review of these continuum models). Here, we only consider models that use a discrete, cell-based approach to describe the behavior and state of single ECs as part of an angiogenic sprout, which enable to capture aspects like sprout branching and anastomosis. Often, discrete modeling of individual cells is combined with continuous modeling of the ECM and diffusing solutes (like VEGF, MMPs and fibronectin), resulting in a hybrid model. Such models have focused among others on the interplay between extracellular signals (either soluble or matrix-bound) and EC behavior to shed light on vascular network formation, and have considered often (tip) cell migration as a result of chemotaxis, haptotaxis and/or haptokinesis. In these terms, taxis means directed movement caused by an external signal, while movement in the case of kinesis is non-directional. Movement can be induced by either soluble or matrix-bound signals (or both), indicated by "chemo" and "hapto" respectively.

In this section we give an overview of hybrid models developed to study the process of angiogenesis, ranging from tip-stalk cell competition and initial EC sprouting to the formation of blood vessel networks at the organ level. The computational methods and the contributions of these models to better understand angiogenesis are discussed. We make a distinction between three groups of models. A first group describes network formation as a result of tip cell migration. These tip cells are represented as points or rigid shapes and their migration is modeled as a reinforced random walk model, with directionality coming from chemotactic and/or haptotactic cues, and sometimes also from ECM topography (contact guidance). The path of the migrating tip cells governs the formation of the network. In a second group of models, a distinction is made between tip cell and stalk cell fate, allowing among others to investigate the role of stalk cell proliferation on sprout growth, but still keeping cell shape constant. Compared to the first group, models of the second group are 


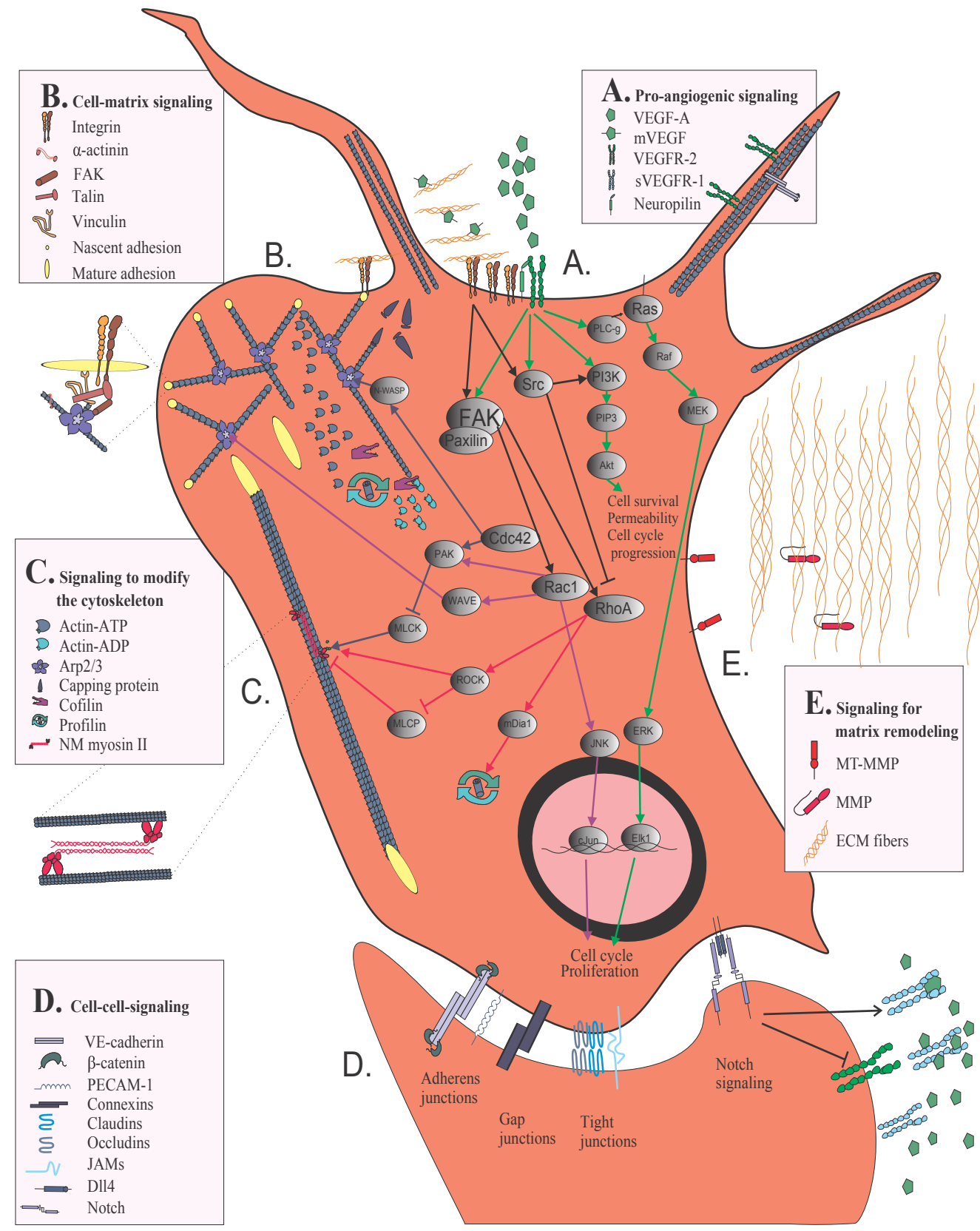

Figure 2: Signaling pathways during angiogenesis: A) Pro-angiogenic signaling. B) Cell-matrix signaling. C) Signaling related to cytoskeletal rearrangements. D) Cell-cell signaling. E) Signaling pathways for matrix remodeling. For more details see Appendix A. 
in general more mechanistic. Finally, in a third group of models, cell shape changes are captured, which enables to investigate the role of cell-cell and cell-matrix interaction in a more refined way, rendering these models better candidates to extend them with cell mechanical principles. Some of the models that we listed in the third group, also incorporate tip cell-stalk cell interaction, meaning that there is some overlap between the second and third group. In fact, the work of Bentley and co-workers, which we list in the third group, has been the first model of angiogenesis that incorporates tip cell selection based on Dll4-Notch signaling [8]. Nevertheless, we list this work within the third group as a prominent example of a model that deals with cell shape dynamics, which from a cell mechanical point of view is a more important criterion for model classification in the context of this review paper. An overview of all models is given in Table 1.

\subsection{Tip cell migration based models}

Many models are based on the assumption that a migrating tip cell sets the path and governs the motion of the whole sprout. Stokes et al. were the first to model chemotactic migration of single ECs in a discrete way [124], by formulating a stochastic differential equation for the rate of change of EC velocity that considered random fluctuations, resistance to motion and a chemotactic bias. They investigated individual cell paths in the presence or absence of the angiogenic stimulus acidic FGF. Simulations, compared with cell paths tracked experimentally by time-lapse videos, showed that EC migration speed is increased by the presence of an angiogenic stimulus, while persistence time is decreased. This led to the suggestion that rate and direction of EC movement might be regulated by different intracellular pathways.

Anderson and Chaplain discretized their continuous tumor-induced angiogenesis model to track individual tip cells during sprouting [2]. Tip cell movement, defined as transition from one grid cell to a neighboring grid cell, was governed by defining a probability for each transition, which was a function of the local concentration (grid cell values) of directional cues found in the extracellular space. The result was a random walk model enhanced with a tumor angiogenic factor (TAF)-based chemotactic and fibronectin-based haptotactic component, combined with (phenomenological) rules for anastomosis, branching and cell proliferation. The model showed that cells affect the chemotactic and haptotactic stimulus by means of TAF uptake and fibronectin uptake and production. The model further suggested that both chemotaxis and haptotaxis are required to obtain a capillary network; chemotaxis was found to be required for the initial outgrowth of the capillary network, while haptotaxis was needed for lateral sprout migration, branching and anastomosis in order to form the typical brush border at the location of the tumor.

In the following years, reinforced random walk models of tip cell migration were also used by others [100, 101, 119]. Sleeman and Wallis used a model similar to Anderson and Chaplain, but used a prescribed TAF and fibronectin distribution $[2,119]$. In 2D and 3D simulations they investigated the relative roles of chemotaxis and haptotaxis in the formation of vascular networks and found that an increase in haptotaxis resulted in more anastomoses, thereby impeding EC migration. Next, Plank and Sleeman developed a lattice-based model of tumor-induced angiogenic sprouting [100]. Tip cells were modeled to migrate based on chemotaxis towards higher concentrations of VEGF and haptotaxis towards - contradictory to earlier models - lower concentrations 
of fibronectin, based on the assumption that a non-degraded ECM serves as a physical barrier for cell migration and that proteolytic fragments of fibronectin serve as a chemoattractant for ECs. Therefore, chemotaxis towards a higher concentration of cell secreted fibronectin protease was also included. Furthermore, at low concentrations of fibronectin protease, EC proliferation increased with protease concentration, implemented as sprout branching at the tip cell position, while a high protease concentration was assumed to lead to EC apoptosis, thereby ceasing further sprout growth. Protease secretion was stimulated by VEGF. The model was used to investigate potential anti-angiogenic strategies. Angiostatin, by deactivating fibronectin protease, resulted in reduced cell migration and thereby prevented the formation of a functional vascular network. Simulations showed that early introduction of angiostatin is most effective in preventing angiogenesis. The removal of the source of VEGF after a certain (simulation) time showed to be less effective in preventing angiogenic sprouting, because VEGF secreted before this time remains present. Finally, in another study from the same group a circular random walk model was used in which cell position was not restricted to the lattice points, but instead cells could move in every direction, resulting in more realistic network shapes [101]. Equations describing chemotactic and haptotactic migration were based on those used by Anderson and Chaplain [2]. Comparison of lattice-based simulations with off-lattice simulations showed similar results. Besides, the model suggested that increased branching and reduced chemotactic sensitivity at high VEGF concentration might explain the brush border effect near a tumor.

Tong and Yuan developed a reinforced random walk model to investigate angiogenic sprouting in the cornea [129]. Sprouting direction was calculated from the direction of the angiogenic factor concentration gradient of the current time step and the migration direction of the previous time step, while randomness was added to represent the influence of the heterogeneous ECM. Sprout growth rate depended on the local angiogenic factor concentration, which was affected by uptake of the angiogenic factor by the cells. With this model they observed that uptake of the angiogenic factor by ECs and its subsequent redistribution by diffusion have significant effects on the structure of vascular networks. A decrease in angiogenic factor uptake led to an increase in both the number of vessels and the movement of the vessel front that was predicted in the cornea.

Milde et al. developed a deterministic hybrid model of sprouting angiogenesis [86]. Tip cells, represented as particles, migrate through the ECM while secreting (fibronectin degrading) MMPs and fibronectin and taking up VEGF. The ECM, consisting of collagen and fibrin, is implemented as a combination of a vector field and a density field, describing the orientation and density of the fibers. ECM fibers bind VEGF (into pockets) and fibronectin, thereby affecting their concentrations and gradients. Tip cell migration direction is determined by fibronectin gradients (haptotaxis), VEGF gradients (chemotaxis) and ECM fiber orientation (contact guidance), while migration speed is determined by ECM fiber density (haptokinesis). Branching was implemented to occur at regions were the matrix structure promoted diverging directions for tip cell migration. This model demonstrated the importance of ECM structure and matrix-bound factors (VEGF and fibronectin) on the dynamics and morphology of generating vascular structures. The structure and density of the ECM showed to have a direct effect on morphology, expansion speed and the number of branches. Besides, an increased number of VEGF pockets and an increased VEGF level per pocket showed to increase the number of branches. 
Owen et al. implemented a chemotaxis (migration towards high VEGF concentration) reinforced random walk model in a multiscale model of vascular tissue growth and blood flow regulated vessel pruning, which is the regression of unnecessary vessels [96]. Simulations showed that strong chemotactic sensitivity leads to fast and directed sprout formation, required for effective network remodeling, while a lower sensitivity resulted in poorer vascularization. Edgar et al. applied a similar reinforced random walk model to microvessels represented as line segments and combined this with stochastic branching and anastomosis in order to investigate the effect of ECM anisotropy on vascular network formation [37,38]. Vessel growth rate was modeled to decay exponentially with matrix density as was observed experimentally, while growth direction was calculated as a function of collagen orientation and local microvessel density. Microvascular structures similar to in vitro experimental results were obtained for various ECM densities and for both an isotropic and anisotropic ECM, with vessels without preferential direction for an isotropic ECM and vessels aligned with the longitudinal ECM fiber direction for an anisotropic ECM. Besides, the model showed that an increase in matrix density leads to a decrease in total vascular length and the number of branches.

Finally, Jain and Jackson investigated the role of VEGF binding in EC migration and capillary sprouting [63]. In their model, chemotaxis was not implemented based on VEGF concentrations or gradients in the ECM, but based on VEGF binding to VEGFR-2 on the cell membrane, in this way providing a first step towards a more mechanistic treatment of chemotactic cues, based on receptor activation. Binding of VEGF to VEGFR-2 and activation of these receptors is described by a system of ordinary differential equations. The number of activated receptors on each of the four sides of a rectangular shaped cell determines polarization and thereby direction of migration. Furthermore, the probability of sprout branching, occurring only at the tip cell, increases with the number of activated receptors up to a saturation level. Simulations were performed of sprouts growing towards VEGF-secreting tumor cells, were the secreted VEGF concentration was varied between simulations. Both the rate of vessel formation and the brush-border effect close to the tumor due to branching agreed well with experiments for these VEGF concentrations.

In other studies, reinforced random walk models of angiogenesis have also been combined with models of tissue regeneration in order to investigate the role of angiogenesis in regeneration (e.g. [22,77]). As this review paper focuses on the modeling of angiogenesis as such, and not on modeling its relation with other processes, we only mention these studies for completeness.

\subsection{Tip cell - stalk cell based models}

In recent years, computational models of angiogenesis have been designed that not only take into account tip cell migration, but make a distinction between tip cells and stalk cells and include other processes like proliferation, maturation and apoptosis, often in a rule-based manner.

Qutub and Popel developed a multiscale model of sprouting at the onset of angiogenesis [105]. In this model that starts with an initial 3D vascular network, ECs in the sprout are represented by means of a leading node and a back node, where the back node of each cell serves as the leading node for the next cell. The distance in between the two nodes can change, in this way accounting for EC elongation. Changes in cell activation, position and growth (change in length as well as 
proliferation) for tip and stalk cells are determined by biologically-based Boolean rules that are coupled to the local VEGF concentration. Tip cell migration depends on the surrounding VEGF concentration (chemotaxis) and the collagen content (haptotaxis), where collagen is degraded by MMPs in a constant way. The migrating tip cell "pulls" on the stalk cells, promoting stalk cell elongation and proliferation, which then "pushes" the tip cell forward. The push-pull system is governed by the set of rules that control the position of the leading and back nodes of the tip and stalk cells, and not by a balance of momentum defined at the cell level, meaning that the importance of mechanics is not yet included in the model. Tip cell formation, proliferation and branching probability were regulated by intracellular Dll4 levels. Compared to previous models, this model not only deals with the effect of extracellular VEGF on tip cell behavior, but also makes a first step towards cell-cell (tip cell-stalk cell) interaction and cell shape changes (change in length), regulated by intracellular molecular signals like Dll4. Simulations showed that the driving force of angiogenesis is a VEGF gradient rather than absolute VEGF concentrations. In a later study this model was adapted and used in a module-based computational platform to investigate exerciseinduced angiogenesis in skeletal muscle [78].

Jackson and Zheng developed a cell based mathematical model of corneal angiogenesis, consisting of a simple mechanical cell elongation model and a biochemical cell phenotype variation model [61]. The tip cell generates a protrusive force, driven by chemotactic gradients and its direction being further modulated by contact guidance of the cell to the surrounding ECM fibers. The protrusive force is transduced to the following stalk cells and is counterbalanced by the adhesion force applied by these cells to the tip cell. The ECs are modeled as spring-dashpot (viscoelastic) systems that can elongate, using experiment-based material properties and dimensions. The elongation is solved from a force balance that takes into account the protrusive force applied by the lamellipodium of the tip cell and the drag between the cell and the ECM (being a first step towards mechanical interactions between a cell and its surrounding ECM). When a stalk cell divides, a new cell is created with an unstretched spring, allowing the tip cell to migrate again. Furthermore, tip cell overtaking as observed by Jakobsson et al. was implemented by the migration of cells from the pre-existing vessels along the sprout to the tip cell position at a constant speed [64]. Cells are simulated as discrete single points representing the leading cell edge, while cellular processes like cell elongation, proliferation and maturation are described with continuous differential equations. With this model they aimed to understand why stalk cell proliferation is required for angiogenic sprouting and why it occurs mainly at the leading edge of the sprout. Nonproliferative sprout extension solely by cell recruitment from the pre-existing vessels showed to be adequate for initial sprout extension, but was insufficient for larger extension due to cell quiescence at the base of the sprout. For the location of stalk cell proliferation, maturation of stalk cells at the base of the sprout by angiopoietins, together with higher availability of VEGF near the tip cell, was suggested as a possible explanation.

Das et al. developed a hybrid agent-field model of sprouting angiogenesis in which individual cells were, based on growth factors and matrix stiffness, assumed to be in either one of four stages: quiescence, proliferation, migration and apoptosis [28]. Intercellular signaling was implemented to occur only through the ECM by secreted molecules like VEGF and MMPs. Initial simulations were performed to compare the simulations with results obtained from experiments using microfluidic 
devices. In a later study, Wood et al. extended this model by making a distinction between tip cells and stalk cells [146]. In this model tip cells do not proliferate, but migrate through the collagen matrix in a 3D fashion requiring degradation of the ECM, their migration direction being controlled by VEGF gradients. Stalk cells proliferate but only migrate in a $2 \mathrm{D}$ fashion in the tunnel created by the leading tip cell. A phenomenological cell-cell interaction force is also considered for stalk cell migration, which, depending on the cell-cell distance, is either an attractive or a repelling force. Furthermore, competition for tip cell phenotype (due to Dll4-Notch signaling; see Figure 2D) is included in a probabilistic way (and based on the distance between neighboring cells). Because of the distinction between tip and stalk cells, processes like sprout breakage, lumen formation and cell phenotype changes could be observed, which was not possible for models in Section 3.1.

Travasso et al. modeled the interface between newly formed capillaries and the ECM with a phase-field model, while tip cells were modeled as agent-based components [130]. With their simulations they demonstrated that tip cell velocity and stalk cell proliferation play important roles in vascular network morphology. An increase in stalk cell proliferation showed to lead to a more branched network constituted by thicker vessels, while a higher tip cell migration velocity leads to a more branched network with thinner vessels.

Finally, Carlier et al. incorporated the intracellular signaling module of Bentley et al. (describing the interplay between VEGFR-2, Dl14 and Notch; see Section 3.3. for a more in depth discussion of the work of Bentley and co-workers) into a hybrid model of angiogenesis during bone fracture healing developed before by Peiffer et al. $[8,18,97]$. ECs were modeled as single agents. Similar to Bentley et al., actin levels of an EC, representing filopodia extension, were modeled to be upregulated when the activated VEGFR-2 level exceeds a certain threshold [8]. Tip cell migration occurs only if the actin level and VEGFR-2 level exceed a certain threshold and speed of the tip cell increases with the number of activated receptors. The direction of migration is determined based on chemotactic and haptotactic gradients, which are calculated from the continuous fields of VEGF concentrations and ECM densities respectively. An increase in extracellular VEGF concentration was found to result in an increased tip cell density up to a certain level, after which a further increase in VEGF concentration downregulated the tip cell phenotype due to mutual inhibition of neighboring cells. The latter result demonstrates the added value of having a multiscale, hybrid model that couples intracellular signaling (VEGFR-2, Dll4 and Notch), individual cell behavior (EC agents) and extracellular information (extracellular, continuous concentrations of VEGF).

\subsection{Cell shape dynamics models}

The computational models of angiogenesis discussed in the previous sections all lack description of EC shape and how this shape may change during sprouting. This makes them less suitable for a more in depth treatment of the role of cell mechanics in angiogenesis, as cell mechanical forces will have a direct effect on cell shape changes. The third group of models captures cell shape dynamics although in most cases without considering any mechanical laws.

A model type that dynamically captures cell shape is the Cellular Potts model (CPM) [5, 12, $29,79,85,114,126]$. This is a lattice-based computational modeling method that allows to simulate the collective behavior of cells in a computationally efficient way, based on energy minimization 
[46]. In every time step the cell surface, represented by connected lattice vertices, is updated probabilistically depending on a set of cell behavior rules (e. g. target cell shape and size) that are translated in an energy change. The likelihood for a certain update depends on the change of the total energy of the system that is associated with the update and is equal to one if the total energy is reduced.

Merks et al. demonstrated that a combination of cell elongation and preferential membrane extension in the direction of an autosecreted chemoattractant suffices to form vascular-like morphologies in a model of vasculogenesis [85]. Bauer et al. were the first to develop a CPM of sprouting angiogenesis [5]. They included processes at the discrete cellular level (migration, growth, proliferation, cellular adhesion, ECM degradation, chemotaxis and haptotaxis) and continuous extracellular level (VEGF diffusion, uptake and decay). A specific aim was to investigate the role of an inhomogeneous stroma (matrix fibers, interstitial fluid and tissue-specific cells) on the sprout formation. To our knowledge, they were the first to come up with a model of angiogenesis that modeled the extracellular space in an explicit way, rather than by means of a continuous field. ECs, matrix fibers, interstitial fluid and tissue-specific cells are all occupying grid cells, interact with each other and compete for space, as captured by appropriate energy terms. They demonstrated that local anisotropies in the stroma influence sprout migration and morphology and might regulate sprout branching and anastomosis. Interestingly, branching and anastomosis occurred naturally as a result of cell-cell and cell-ECM interactions, and not as a consequence of pre-imposed (phenomenological) rules. Furthermore, the presence of steep VEGF gradients due to release of matrix-bound VEGF isoforms led to narrower sprouts than the presence of only a shallow gradient. It was also demonstrated that the region at which EC proliferation takes place does not affect sprout morphology, but does influence sprout migration speed. Mahoney et al. used this model to search for new strategies to disrupt angiogenesis induced by tumors [79]. They added a continuous oxygen model consisting of a reaction-diffusion equation describing oxygen diffusion, degradation, secretion by ECs and absorption by tumor cells. A flow model was added that estimates blood flow through the newly formed network and determines the location of oxygen secretion. By investigating the amount of oxygen provided by the newly formed network to the tumor, various new angiogenesis-blocking therapies were explored in silico. Shirinifard et al. developed a CPM of a growing tumor within a vascular network [114]. As the tumor grows, the oxygen level drops and tumor cells secrete pro-angiogenic factors. ECs in existing vascular structures are activated by these factors and migrate chemotactically toward the tumor. In this way the effects of angiogenesis on tumor growth was investigated. Szabó and Czirók investigated the process of multicellular sprout elongation from cellular aggregates without chemotactic and haptotactic factors [126]. They demonstrated that migration of only a tip cell, pulling along the other cells via cell-cell adhesions, is not sufficient for sprout elongation. Instead, active cellular motility of the stalk cells, guided by cell-cell contacts, and a preferential attraction to surfaces of elongated cells is required. Boas et al. developed a CPM to simulate angiogenic sprouting in a fibrin matrix, corresponding to an in vitro setup that explored EC invasion from a monolayer [12]. ECs, fibrin matrix and a basement membrane in between the EC monolayer and the fibrin matrix were explicitly modeled by means of the CPM. ECs could secrete proteolytic enzymes (MMP and u-PA (urokinase-type plasminogen activator), their dynamics being captured by a partial differential equation) that degraded the 
matrix and were guided by haptotactic cues. By changing the amount of secretion of both MMP and u-PA, structures were obtained ranging from sprouts to cyst-like structures and monolayers. Daub et al. investigated the role of the ECM in coordinating cellular motility during angiogenic sprouting [29]. The ECM was implemented as a continuous field using a partial differential equiation. ECM degradation was implemented by VEGF-induced MMP-secretion. Cell motility was regulated by the absolute ECM concentration (haptokinesis) and by gradients in ECM concentrations (haptotaxis). In exploratory simulations it was shown that these implementations suffice to obtain angiogenic sprout formation. Furthermore, it was shown that faster ECM degradation leads to a less compact network with bigger sprouts, while slower degradation has the reverse effect. Finally, although they did not focus on angiogenesis, we want to mention the work of Köhn-Luque and co-workers. They developed a CPM model of vascular patterning during early embryonic vasculogenesis [71]. Besides chemotactic migration towards higher concentrations of soluble VEGF molecules, cells were also assumed to produce ECM constituents that bind VEGF molecules, resulting in a stronger chemotactic migration towards high concentrations of ECM-bound VEGF molecules compared to soluble VEGF molecules. With this model vascular networks comparable to experimentally obtained networks were observed. Thereby, this model supports the suggestion that the ECM plays an import role in the paracrine regulation of vascular pattern formation.

In the CPM models described above, the rules imposed to govern cell shape changes did not account for cell or matrix mechanics in an explicit way. Van Oers et al. developed a hybrid model to describe the behavior of single ECs and multicellular systems on compliant ECMs by combining a CPM, which describes EC motility, and a finite element (FE) model to calculate cell traction force-induced ECM deformation [133]. A phenomenological model of traction force distribution based on Lemmon and Romer is assumed, leading to forces that are directed towards the cell center and that increase with distance to the cell center such that highest traction forces are applied by the protruding areas of the cell [76]. The Hamiltonian accounting for the total energy of the system was extended with an ECM strain-dependent term that favors cell extension in the direction of principal strain, in this way capturing to some extent durotaxis (by assuming that principal strain directions will coincide with directions of highest ECM stiffness due to strain stiffening). These rules together lead to a mechanical cell-ECM interaction in which cells affect their own behavior by straining the ECM. Simulations demonstrated the alignment of single cells and cell pairs for intermediate, but not high and low ECM stiffnesses, similar to experimental observations. Besides, the model was able to reproduce network formation starting from a uniform cell distribution and sprouting from endothelial spheroids, thereby presenting cell-ECM mechanical interactions as an alternative mechanism for controlling single and multiple cell behavior, relevant for angiogenesis.

Although some of the models of sprouting angiogenesis described so far make an attempt to capture cell-cell interaction between neighboring ECs within the same sprout, the (molecular) mechanisms that control these interactions are to a large extent lacking. Bentley and co-workers were the first to design detailed agent-based models of cell-cell interactions, governed by Dll4Notch signaling. In a first model, a vessel was represented as a cylindrical capillary composed of 10 cells, where each cell consisted of multiple membrane agents [8]. A periodic boundary was applied to ensure that every cell has two neighboring cells. A (fixed) uniform or gradient VEGF concentration was prescribed extracellularly. In each time step, VEGFR-2 activation levels, and 
thereby Dl14 level, were calculated for each membrane agent based on local VEGF concentrations. Dll4 activates Notch in membrane agents from neighboring cells, thereby downregulating the VEGFR-2 level. In this way, tip cell selection occurs by lateral inhibition. A filopodium can extend from a membrane agent if sufficient actin is recruited. The formed filopodium can then extend or retract based on the actin level. The filopodia were hypothesized to enhance the lateral inhibition between tip and stalk cells since their formation, which is favored for tip cells, increases the cell surface and thereby VEGF uptake by the cell. Various simulations showed that this model is able to predict the salt-and-pepper pattern of stalk and tip cells that is also observed in vivo. Furthermore, it was shown that a VEGF gradient leads to a faster stabilization of tip-stalk cell distribution than a uniform VEGF field. In a study by Jakobsson et al. the model was adapted by creating a sprout containing a tip cell and multiple stalk cells, which was achieved by removing the periodic boundary conditions used before [64]. After the typical salt-and-pepper pattern of tip and stalk cells was formed, cells were allowed to move towards the tip of the sprout by switching position with their neighbor cells. The switching probability for each cell was calculated based on VEGFR2 and Notch activity. Simulations, compared with experiments, showed that the dynamic position shuffling of ECs in a sprout is negatively regulated by Notch activity. In another study, Bentley et $a l$. adapted their original model by connecting the membrane agents with Hookean springs to include actin cortical tension [10]. This allowed the authors to add cell migration and thereby sprout formation to their model. Filopodia formed by tip cells are modeled as a chain of spring-connected agents, allowing them to bend, grow and retract in discrete steps. Filopodia growth direction was made dependent on the local VEGF level. Cell migration was implemented by pulling the cell body along the extended filopodia. Simulations showed that anastomosis of two tip cells leads to a flip in cell fates. Due to lateral inhibition, one tip cell will dominate over the other, which then becomes a stalk cell. This change directly affects the fate of other neighboring cells, showing the dynamic nature of the tip-stalk cell partition [10]. In yet another follow-up study, the role of cellcell adhesion dynamics behind this rearrangement of cells in a growing sprout was investigated more in depth [7]. In this model, cells were not allowed to extend from the base vessel, but instead cells were allowed to pass each other by migrating over the cylindrical mesh. The authors created a hybrid model, in which the membrane agents that represent the membrane and actin cortex, are coupled to a CPM of the cell body allowing local adhesion dynamics between cells to be incorporated. At every time step, the junction between two cells is remodeled by so called copyflips that lead to protrusion of the membrane of a cell at the expense of another cell. The probability of these copyflips is regulated by Notch levels and sprout direction. Two mechanisms for cell rearrangement were proposed: differential adhesion and differential polarized junctional-cortex protrusions. In the first mechanism, cells with higher levels of activated VEGFR-2 are weakly adhesive representing endocytosis of VE-cadherin away from the junction, while Notch-inhibited cells are more strongly adhesive. In the second mechanism, junctional protrusions by copyflips are more likely to occur for cells in which Notch-signaling is low and/or junctional protrusions are only allowed if made in the same direction as the growing sprout. Simulations validated by experiments showed that both Notch-regulated cell-cell adhesion strength and Notch-regulated junctional protrusions are required for simulating in vitro cell rearrangement. 


\section{Role of cell mechanics in angiogenesis}

As already mentioned in the introduction, we want to verify to what extent current models of sprouting angiogenesis deal with cell mechanical principles in an explicit way. Before we do this, we will first highlight a number of experimental findings on adhesion and cytoskeletal dynamics that demonstrate the importance of cell mechanics during sprouting angiogenesis, in particular for the role of migration in a growing sprout. We will also briefly describe a number of generic (molecular mechanical) mechanisms of single cell migration, as this will help us later on in understanding computational models of single cell migration (Section 5.), as well as to make recommendations for combining computational models of angiogenesis and cell migration into mechanochemical models of angiogenesis (Section 6.).

\subsection{Experimental observations}

Recent studies have demonstrated that mechanical properties of the ECM affect the angiogenic response of ECs [26,70,75,80,84]. During sprouting, the mechanical coupling between neighboring ECs on the one hand and between the ECs and their extracellular environment on the other hand needs to be orchestrated to allow a strictly controlled outgrowth of the sprout without disintegration of the vessel. Beside the mechanical coupling between the cells and their surroundings, change in cell shape and movement must be the consequence of forces acting upon the cell, following the laws of classical (Newtonian) mechanics. Cells generate internal contractile (traction) and protrusive forces with their cytoskeleton and are able to transduce them to the extracellular environment through focal adhesions [136]. For readers who are less familiar with cytoskeletal components and their involvement in the generation of protrusive and traction forces, an overview is given in Appendix B.

RNA analysis of invading ECs at different timepoints showed that the molecular profile of ECs during invasion of three-dimensional collagen matrices typically displays a downregulation of genes associated with tight junctions, whereas a set of adhesion molecule genes involved in adherens junctions, ECM interactions and proteases is upregulated [125]. Cell-cell junctions become partially disorganized to allow ECs to sprout [20,33]. When the new vessels mature and stabilize, junctional integrity is re-established. In quiescent ECs VE-cadherin promotes vessel stabilization by inhibiting VEGFR-2 signaling [20]. Integrins have been shown to play an important role in angiogenesis $[90,110]$ and specific integrins that are upregulated during angiogenesis include $\alpha \mathrm{v} \beta 3$, $\alpha \mathrm{v} \beta 5, \alpha 1 \beta 1, \alpha 1 \beta 2, \alpha 1 \beta 4, \alpha 1 \beta 5$ [116]. Proteases needed to degrade the matrix are upregulated as well. Taken together, these effects will change adhesional mechanics of ECs with respect to neighboring cells and the ECM, as well as ECM mechanics. In turn it will affect the cells ability to transfer forces to the extracellular environment, and therefore its ability to change shape and migrate.

The groups of Waterman and Gardel studied directional control of EC morphogenesis and movement. Based on their experimental data, they propose a mechanism of myosin II regulation of directed EC migration in 3D ECM environments [40]. When a sprout is navigating and elongating into a partially degraded ECM, tip cell extensions were observed to initiate where sites of nascent 
lamellipodia coincide with transient local depletions of non-muscle (NM) Myosin IIB. This local depletion would briefly allow the extensions to escape from cortical tension. For the extension to change EC migration direction, the lamellipodium is then presumed to encounter and adhere to more distal ECM that is stiff enough to re-establish actomyosin contractility for movement in the new direction [40].

Apart from actomyosin dynamics, microtubules play a role as well. Elongation of ECs during angiogenesis has been observed microscopically and appeared to be accompanied by stabilization of microtubules and their alignment into parallel arrays directed at the growing tip [52]. While these observations underline the role of the cytoskeleton for EC shape changes and migration during sprouting angiogenesis, cytoskeletalon organization also seems to be correlated to the orientation of division, again adding to the directionality of sprout growth. In retinal vessels in vivo, the plane of endothelial cytokinesis was oriented perpendicular to the vessel long axis [149]. In this way, the division appears orchestrated for the daughter cells to contribute to the elongation of the sprout. Experimental data suggests that orientation for proliferation is regulated by VEGF signaling during blood vessel formation [149]. Next to chemical signals, the spatial distribution of the adhesive contacts and external forces that cells encounter also influence the molecular composition of the cell cortex, indirectly affecting spindle orientation and daughter cell positioning [128]. This demonstrates how the crosstalk between actin, microtubules and adhesion complexes is critical to division and its contribution to sprouting.

In summary, these experimental observations demonstrate that sprouting angiogenesis involves important changes in the local cell mechanical environment that have to do with cytoskeletal mechanics (actin, myosin, microtubules), adhesional mechanics of ECs with respect to neighboring cells and the ECM, as well as ECM mechanics. These changes will modulate the cells ability to generate internal forces and to transduce them to neighboring cells or the ECM, in turn affecting its ability to change shape and to migrate.

\subsection{Cell mechanics in computational models of angiogenesis}

From Section 3.1. we can learn that none of the tip cell migration based models consider cell mechanical forces in the equations that govern tip cell migration. Movement of the tip cell is described globally by chemotactic, haptotactic and contact guidance cues, but the underlying mechanisms are not considered. Besides, the ECM is treated as a continuous field, making it impossible to describe cell-ECM mechanics in a mechanically sound manner.

If we look at the tip-stalk cell models in Section 3.2., some of the models make a first step towards integration of cell mechanical principles in computational models of angiogenesis, albeit in a simplified way, as cell shape changes are either not considered or limited to a change in length. Qutub and Popel model pulling of the tip cell on the following stalk cells by tip cell migration and pushing of the tip cell by stalk cell proliferation [105]. The existence of such a push-pull system is still under debate. As described in Section 2., a dynamic interchange between tip and stalk cells has been observed experimentally $[3,64]$, leading to overtaking of the tip cell by other cells in the sprout. Besides, in the work of Qutub and Popel a change in cell length does not follow from a momentum balance but from a set of non-mechanical rules, so that strictly speaking pulling and 
pushing cannot be interpreted here in a mechanical sence. Jackson and Zheng use a force balance to describe cell-cell mechanics in a sprout, including a protrusive force applied by the tip cell, cellcell adhesion and cell-ECM friction [61]. EC mechanics is still very simple though and limited to a 1D spring-dashpot system. Finally, Wood et al. consider a phenomenological cell-cell interaction force to modulate stalk cell migration velocity [146].

Including cell shape in models of angiogenesis as described in Section 3.3. allows for a more extensive implementation of single cell mechanics. Bauer et al. were the first to model the extracellular environment of an angiogenic sprout in an explicit way, using a CPM [5]. In their model, cells had to degrade or migrate around extracellular obstacles. Although this model does not include cell mechanics or cell-ECM mechanical interaction, it shows that inhomogeneous ECM can play an important role in processes as branching and anastomosis. Van Oers et al. were the first to introduce cell and matrix mechanics in a more explicit way and demonstrated that cell traction force-induced matrix strain and its effect on single cell shape can be important for multicellular organization, such as during sprouting angiogenesis [133]. Cell traction forces were described in a phenomenological way, without considering the governing cytoskeletal or adhesional mechanics. In addition, cell shape changes do not follow from a balance of momentum, but instead a straindependent term was added in the Hamiltonian, in this way leading to an implementation of cell mechanics that is only weakly based on mechanical principles.

The introduction of Hookean springs that interconnect membrane agents allowed Bentley and co-workers to have a first approximation of the role of actin cortical stiffness in the change of cell shape, by calculating position updates of non-adhering membrane agents from a static equilibrium of nodal forces [10]. Values of spring constants were optimized to make sure obtained morphological curvature of tip and stalk cells were realistic, without any attempt to relate them to measured (endothelial) cell mechanical properties. Filopodium extension and retraction was governed by local (at the level of the membrane agents) actin and adhesion dynamics. As to the latter, the adhesion state of membrane agents was allowed to change between non-adhering and fully adhering (fixed in space), based on a number of non-mechanical rules (related to local actin levels and position within and length of the filopodium, as well as the presence of filopodia of neighboring cells). While their implementation is to a large extent neglecting the underlying cell and molecular mechanical principles and forces that can be associated with actin protrusion and adhesion, and while the model did not account for cell-ECM mechanical interaction (as only a field of VEGF concentration was considered), it is an interesting first attempt to couple local molecular signaling events to cellular forces that together govern dynamic cell shape changes during sprouting angiogenesis. In their latest model, Bentley et al. coupled the membrane agent model to a CPM [7]. In this model cells were represented by membrane agents in a cylindrical shape, similar to [8] and [64]. Although this model does not capture 3D cell shape changes and creation of a new sprout by migration of cells out of a vessel as was modeled in [10], the coupling of membrane agents to a CPM enabled to model dynamic positioning of cells in a sprout. This dynamic positioning is modeled by, from a mechanical point of view incorrect, transfer of membrane agents between cells. This allowed them to study the role of VE-cadherin and adherens junctions on connectivity in a sprout. By regulation of cell-cell adhesion and membrane protrusion by Notch signaling and VEGFR-2 activation, this model forms the first and only computational model of angiogenesis that couples 
molecular signaling to subcellular mechanical processes.

\section{Computational models of cell migration}

From the previous section it has become clear that cell mechanics is often overlooked in computational models of angiogenesis. Cell migration is often prescribed based on chemotactic, haptotactic and/or contact guidance cues, while cytoskeletal and adhesional mechanics that govern cell migration are not considered. By neglecting cell mechanical principles, forces and the mechanical laws they need to respect, model solutions may be obtained that are not valid from a mechanical point of view. In this section we will review computational models of single cell migration that have incorporated cell mechanical processes. By extending computational models of angiogenesis (as reviewed in Section 3.) with cell mechanical models of single cell migration, one can try to resolve the limitations of current angiogenesis models that were addressed in Section 4. (and that will further be discussed in Section 6.). Models of cell migration can be divided broadly into two groups: models that aim at predicting cell shape dynamics based on a continuum description of cytoskeletal remodeling and activity, and models that use discrete descriptions of the cytoskeleton. Only models that contain both a description of cell shape and intracellular processes are discussed here. An overview of these models is given in Table 2. It should be noted that the aim of this section is to give an insight in the integration and coupling of biochemical and mechanical subcellular processes in models of cell migration, rather than to give a complete overview of cell migration modeling. For a more complete overview of computational modeling on cell shape and motility, the reader is referred to some prominent review papers in the field $[27,55,106,141]$.

\subsection{Continuum representation of the cytoskeleton}

Models of the first group define a dynamic shape of the cell and predict how the cell deforms and moves based on internally generated forces. Actin polymerization at the leading cell edge and actomyosin contraction in the cell body lead to membrane protrusion and retraction respectively. Various methods have been used to model cell shape dynamics.

Vanderlei et al. developed a model of cell migration and polarization using the immersed boundary method, which allows capturing actual forces and deformations of the cell membrane [135]. A mechanical cell model consisting of an elastic cell boundary and a viscous cytosol is combined with a biochemical model of the actin cytoskeleton. The effect of actin polymerization in the cell is directly converted into protrusion forces applied to the cell membrane. Together with an elastic cell cortex these forces describe cell deformation. The biochemical model describes diffusion and advection of Rho GTPase in active and inactive forms, where actin protrusion occurs in regions with the highest active form concentration. Simulations without intracellular biochemical regulation show that the properties of the cortex and the strength of actin-generated protrusive force determine the cell shape and can explain why different shapes are observed for various cell types. Higher protrusion forces lead to more crescent-shaped cells. Including also the biochemical systems, more stable cell shapes were obtained. 
Taber et al. developed a mechanical model of 1D and 2D keratocyte crawling on a flat substrate, including actin polymerization and frictional adhesion at the leading edge and continuous contraction in the direction of cell migration [127]. The cell model is numerically implemented by means of the FE method as a porous elastic material saturated with a viscous fluid. From simulations with the 1D model it was observed that cell speed depends on adhesion strength in a biphasic manner as was seen in experiments before for other cell types. Both at low and high adhesiveness cell migration is slow due to respectively the inability to gain enough traction and the inability to release from the substrate. Cell migration speed is highest at intermediate adhesion strengths. Besides, a forward motion of actin at the leading cell edge and at the rear of the cell was obtained, while retrograde flow took place in the middle of the cell as was observed before in experiments. In the $2 \mathrm{D}$ model, traction forces applied to the substrate were investigated and showed to be largest at the leading and rear edge of the cell as well as near the sharp corners of crescent cell shape. However, the model predicted an oscillatory cell shape, while keratocytes are known to move with a nearly constant shape.

Shao et al. developed a model of cell motility combining actin flow, cell adhesion and morphology [112]. The phase-field method is used to describe the moving cell boundary. This phase field is coupled to local actin flow velocity. The actin network is modeled as a viscous fluid which deforms due to a contractile stress generated by actomyosin contraction and an expansive stress generated by actin filament polymerization at the leading cell edge. Together with surface tension and membrane bending force, these stresses determine motility and deformation of the cell. Discrete adhesions binding the actin network to the underlying substrate are formed at locations of high actin concentration. Upon relative movement of the actin network to the substrate, the adhesions apply forces to the actin network representing the actin-substrate binding. Initially, adhesion forces are modeled as elastic forces. Upon flow of the actin network, adhesions become stretched and break, after which the adhesive force is modeled as a drag force. Adhesions are removed at a constant rate and are directly replaced by new adhesions. Finally, reaction-diffusion equations, containing advection and diffusion terms, are included to describe the dynamic distribution of actin and myosin concentration in the cell. In simulations the cell started to migrate on a rigid substrate upon breaking of symmetry for the actin concentration, and showed a fanlike cell shape and retrograde flow of actin at the front region of the cell comparable to experimental data. Further, by varying the myosin concentration and adhesion strength different cell shapes similar to experimental observations were obtained. In a later study, Camley et al. extended this model to describe cell motility on uniaxial micropatterned substrates [16]. In this model, protrusive stress is allowed only at locations were the cell adheres to the substrate. On an adhesive stripe the simulated cell showed periodic migration due to regular depolarization of the cell.

Also Ziebert et al. used the phase-field method to model the cell boundary dynamics [151]. They combined it with a vector field describing the orientation of the actin filaments throughout the cell. Intracellular processes like actin polymerization, actomyosin contraction and actin bundle formation, described in a partial differential equiation together with area conservation, drive the cell boundary remodeling dynamics. At the membrane, orientation of actin is created perpendicular to the membrane representing actin polymerization, while the orientation is decreased at the same time due to depolymerization and diffusion. The orientation vectors are used to calcu- 
late the advection of the phase field due to actin polymerization and the effect of tensile stresses on the membrane due to actomyosin contraction. The model successfully predicts cellular motion and various keratocyte-like shapes that agree with experimental observations. In a follow-up study the model was extended with dynamics for adhesion site formation and substrate compliance [150]. The force applied by actin filament propulsion was made dependent on the number of adhesive contacts. The substrate is modeled as an effective spring, determining breakage of adhesions. Simulations are performed with various adhesion patterns on the substrate, effecting adhesion formation in the cell. Cells are shown to migrate along alternating stripes of low and high adhesiveness. Further, besides steady cell migration, also periodic propulsion-relaxation migration is obtained. Cell speed increases as more adhesions are formed. Due to the increased force applied to the substrate, substrate deformation increases, resulting in adhesive breakage. As a result, the speed drops, allowing new adhesions to form.

Wolgemuth et al. used the level set method for moving boundary simulations to investigate four different mechanisms for fish keratocyte shape formation and migration [144, 145]. In a first model, actin polymerization occurs at the cell membrane, except for a region at the rear of the cell where depolymerization takes place. Diffusion of G-actin from this region to the locations for polymerization limits migration speed. Second, the transport of membrane vesicles over microtubules from the rear of the cell to the front, in order to create space for actin protrusion by fusion of the vesicles with the membrane, is implemented. Here, cell migration speed is limited by the density of microtubules. In a third model, distribution of Rac and Rho are simulated, resulting in regions of actin protrusion for high Rac concentration and stress fiber formation and contractility for high Rho concentration. The model is similar to, but simpler than that of Marée et al. that will be discussed in a next paragraph [82]. In a fourth model, actomyosin contraction is added. The cytoskeleton is modeled as a viscous fluid. Polymerization rate at the membrane is decreased with an increase in myosin concentration because myosin acts to bundle actin. Also protrusion rate related to the normal of the membrane curvature and Rac and Rho distribution are investigated. With these different models it was shown that actin protrusion, actomyosin contraction and cell adhesion are not required altogether for cell migration, but that combinations of some of these processes can already lead to a realistic description of keratocyte shape and migration.

Herant and Dembo developed a low-Reynolds number hydrodynamic FE code called Cytopede to investigate 3D migration of cells on flat substrates $[49,50]$. The cell geometry is modeled with a FE method as a single layer of 12-node elements, while cytosol and cytoskeleton are modeled as a two-phase fluid and described with continuum mechanics at the mesoscopic scale. Equations of mass and momentum conservation of these constituents determine the motion of the cell and cytoskeleton flow within the cell. Constitutive equations and boundary conditions are prescribed to mimic biological aspects of cell migration. Migration is initiated by local release of a polymerization messenger at the cell membrane. This messenger stimulates polymerization of the cytoskeleton, leading to membrane protrusion. At locations of polymerization, the membrane is pushed outward, while the cytoskeleton is pushed inward. A compensatory force is applied to the protruding membrane to prevent it from bulging upwards. Membrane that comes into contact with the substrate adheres to the substrate with a no-slip condition. In this model, cytoskeleton contraction by myosin is omitted. After initial cell attachment and flattening on the substrate, stimulation 
of network polymerization at a selected part of the circumference of the cell leads to cell migration. By changing the fraction of cell perimeter where polymerization takes place, typical cell shapes during migration were obtained for keratocytes and fibroblasts [50].

Marée et al. investigated the effects of feedback between phosphoinositides (PIs) and Rho family of GTPases on cell polarization and migration [82]. They developed a computational model, based on earlier work of Marée et al., Dawes and Edelstein-Keshet and Jilkine and EdelsteinKeshet, integrating the signaling pathways of Rho family of GTPases and PIs with a set of partial differential equations, and the cytoskeletal dynamics with a CPM [31, 65, 83]. F-actin filaments are placed on a hexagonal grid, which allows approaching the $70^{\circ}$ angle between filaments due to ARP2/3-mediated branching. Actin filament polymerization through Arp2/3 activation is stimulated directly by Cdc 42 and indirectly through PIs by Rac. When actin filaments reach the edge of their grid they start to apply a force to the edge promoting membrane protrusion. On the other hand, presence of Rho stimulates membrane retraction through actomyosin contraction. The performed simulations demonstrated that feedback between the family of Rho GTPases and PIs is required for communication across the cell resulting in a global decision for cell polarization. Besides, it was shown that cell shape deformation possibly provides an extra feedback to cell polarization.

Stéphanou et al. developed a mathematical model of random fibroblast migration as a result of the cyclic repeat of membrane protrusion, adhesion to the substrate and cell movement via generated traction forces [122]. For membrane protrusions, a model previously developed by Alt and Tranquillo is used that describes the molecular events of actin turnover dynamics and mechanical properties of the cell membrane and the cytoskeleton [1]. A hydrostatic pressure based on actin density and actin polymerization leads to membrane protrusion. partial differential equiations are used to describe actin conservation and force balance. Maturation of cell adhesions is implemented by using three different adhesion types: adhesion point, focal complex and focal adhesion. Adhesions can mature based on experienced traction forces. Each adhesion increases the friction between the cell membrane and underlying substrate, while only matured adhesions add to membrane protrusion. The probability to form a new adhesion increases with the membrane extension size and the amount of actin. Stress fibers are formed between the focal adhesions and the cell centroid and contractile forces lead to translation of the cell centroid. In simulations of cell migration on substrates with various adhesion strengths, the experimentally observed biphasic relationship between cell speed and adhesion strength was obtained. In a later study, the cell adhesion formation, maturation and fate were coupled to actin fiber remodeling [41]. When an adhesion point has reached a certain size it matures into a focal complex. This causes the membrane to become fixed to the substrate and promotes the formation of a dorsal fiber, growing in radial direction towards the cell center, and the spontaneous formation of a transverse fiber, connecting the focal complex with neighboring focal complexes. When the dorsal fiber connects to the contractile circular bundle in the lamella, the focal complex matures into a focal adhesion and the dorsal fiber becomes a contractile dorsal stress fiber and the transverse fiber a contractile transverse stress fiber. The transverse stress fiber contracts, leading to maturation of the neighboring focal complexes. When the contractile forces applied by dorsal and transverse stress fibers on a focal adhesion drop below a certain threshold, the focal adhesion and connected stress fibers disassemble. Cell migration on substrates with square adhesion patches with different interpatch distances was simulated. Short 
interpatch distances lead to stationary states, while larger interpatch distances lead to partially constrained cell states with oscillating free membrane sections. The interpatch distance showed to be more important for the adhesion maturation process than cell contractility. Further, a biphasic relationship between generated traction force and interpatch distance was observed, with highest traction forces for intermediate interpatch distances, independent of cell phenotype. The adhesion lifetime and growing rate of the actin fibers showed to be the limiting parameters of cell morphology and stability.

Finally, over the last decade several models of 2D lamellipodium dynamics have been designed $[17,35,47,87,107,108,142]$. A few of these models coupled lamellipodium dynamics to cellular shape and deformation. However, since the focus of these models is primarily on describing cell shape, we will not discuss them any further here.

\subsection{Discrete representation of the cytoskeleton}

Based on various actin and myosin dynamics, the models described in the previous section are able to describe realistic cell shapes and motility. Lamellipodium protrusion and stress fiber contraction are implemented as stresses applied to a continuous actin cytoskeleton or as forces applied directly to the cell membrane. In a different group of models described below, the cytoskeletal components are implemented in a discrete way.

In several studies discrete cytoskeletal dynamics have been implemented to simulate active cell adhesion and migration [4,67,68]. Barreto et al. included prestressed actin bundles and microtubules, cytoplasm, a nucleus and an actin cortex in a 3D FE model [4]. The cytoskeletal components are implemented based on the principles of tensegrity, assuming that microtubules resist both compressive and tensional forces and actin bundles only tensile forces. Both components are allowed to move independently of each other. The mechanical role of each intracellular component is investigated by performing simulations of cell compression with a spherical bead on cells in which intracellular components are selectively removed. Simulations were compared with results from AFM experiments. It was shown that the microtubules and the actin cortex are vital to maintain cell force and rigidity. Kim et al. designed a computational cell model to investigate cell migration behavior on a 3D curved surface [67]. Haptotactic migration is simulated by covering the surface with a gradient in ligand density. Focal adhesion dynamics, cytoskeletal and nuclear membrane remodeling and actin motor activity are incorporated into the model. In their model the cell is represented by an inner and an outer membrane, representing the nuclear and cellular membrane. Nodes on the cellular membrane represent clusters of integrins. Upon binding of these integrins to ligands on the ECM, various force vectors act on the respective node: force due to frictional dissipation (caused by rupturing of neighboring FA-substrate ligand bonds), force due to stretching of the focal adhesion complex (only for cellular membrane nodes), elastic force resulting from membrane stretching and a force generated by actomyosin contraction. Special emphasis is on modeling the integrin-ligand bond formation and rupturing for various surface curvatures and on contractile forces generated by stress fibers. Simulations of cell migration in rectangular conduits and circular lumens with different widths showed a similar relationship between conduit width and cell migration velocity as was obtained experimentally. In a second study, the effect of 
ligand surface density on cell migration speed and the adhesion on various $2 \mathrm{D}$ fibronectin-coated surfaces were investigated [68]. An extra constant force vector representing protrusive force applied by the lamellipodium was applied to the cellular membrane nodes on the leading edges of the cell. A cell migration speed versus ligand surface density relationship similar to experiments was obtained and high stress concentrations at sharp geometrically patterned edges were observed.

\section{Discussion}

In the last two decades, various models of angiogenic sprouting have been designed. Many of these models have focused on vascular network formation, and how network dynamics and morphology is affected by extracellular signals, which can be soluble or matrix-bound. These models have shown good resemblance with experimental observations, for example in terms of vessel density, sprouting velocity and formed network structures. Besides EC proliferation and elongation, sprout growth in these models relies on tip cell migration by processes as chemotaxis, haptotaxis, haptokinesis and/or contact guidance, and these models have been complemented with descriptions for branching, anastomosis and /or proliferation. By performing in silico experiments on the effects of ECM density and degradation, VEGF concentration and gradients, and ligand distributions, they have increased our understanding on the importance of microenvironmental factors for sprout growth and vascular network formation. Despite these merits, a number of shortcomings can be discerned, which we have translated below into a number of recommendations for future work.

\subsection{Towards angiogenesis models that better capture cell-matrix mechani- cal interactions}

Because cell migration is an essential ingredient of sprouting angiogenesis, we have described the underlying cytoskeletal changes during cell migration as well as some of the molecular players that govern these changes in Appendix A and B. As mentioned in Section 4.1. the study of mechanical forces is as crucial for understanding cell migration as the study of these molecular players, for the simple reason that a moving cell must obey the fundamental laws of classical mechanics. This means that cell motility or shape changes should arise from a proper equation of motion (force balance) that is imposed at the relevant length scale (which can be either cellular or molecular, depending on the research question). By adopting mechanical equations as an integral part of computational models of cell migration and/or angiogenesis, one could effectively restrict the solution space to those solutions that are mechanically sound and feasible. In addition, by having mechanical model variables, such as forces exerted by cells on their ECM, one has the ability of validating not only the 'biological', but also the 'mechanical performance' of the model, in this way contributing to model fidelity. As already mentioned in the introduction, advantages of mechanochemical models that integrate mechanical and biological variables have already been explored by Oster, Murray and others, although at that time at the tissue scale. For a more extensive 
discussion on this topic we refer to [88] and [134].

As was discussed in Section 4.2., only a few computational models of angiogenesis include simple mechanical equations which could be seen as a first step towards a more elaborate treatment of cell-matrix mechanics, making it an aspect that so far has received little attention. At the same time it means that there is an opportunity to improve these models by extending them with a proper description of cell and matrix mechanics. By recovering EC shape changes and movements as well as ECM deformation from classical mechanics, one could ensure that predicted behavior is physically (mechanically) sound.

As described in Section 5. of this review, models of single cell migration containing detailed descriptions of the dynamics of cytoskeletal components and cell shapes have already been developed $[4,16,41,49,50,67,68,82,112,122,127,135,144,145,150,151]$. In these models one or more of the force generating and/or transducing components of the cell's molecular mechanical machinery, such as the lamellipodium, focal adhesions and stress fibers, either or not in conjunction with signaling molecules that modulate their dynamics [135], are included. These models all capture to some extent the mechanics of a single, migrating cell. Some models describe force generating processes such as actin polymerization and stress fiber contraction and apply the corresponding forces as acting on the cell membrane and actin cortex. The membrane then deforms based on these forces and based on membrane tension and bending stiffness. Based on these implementations these models are able to come up with reasonable cell shapes and migration modes with respect to experiments. At the same time, all these models together also show that there is not one migration mechanism that applies to all cells and circumstances. If we look at the mechanisms (membrane protrusion and actomyosin contraction) and components (lamellipodia, stress fibers and adhesions) included in the variety of cell migration models, then apparently none of these are indispensable for cell migration (see Table 2). This agrees with a conclusion that was drawn recently by Lämmermann and Sixt [74]. Based on migration modes and mechanisms observed for various cell types, they stated that the method of migration is determined by the balance between adhesion, contraction and actin polymerization. By changing the balance between these mechanisms, migration techniques ranging from blebbing motility to actin-polymerization based motility can be obtained.

In order to address the lack of a proper description of cell-matrix mechanical interaction in the current computational models of angiogenesis, one could think of extending an angiogenesis model (as reviewed in Section 3.) with a cell migration model that is based on mechanical principles (as reviewed in Section 5.). A concrete suggestion of such a combination of models is given in Section 6.3. However, before integrating mechanical principles from cell migration models in models of angiogenesis, a number of remarks should be made.

First of all, Rangarajan and Zaman stated in a recent review paper that one of the main limitations of current cell migration models is the lack of models describing cell migration in 3D [106]. Current models describe migration on a flat 2D substrate, where cells show lamellipodium driven cell migration. However, cell migration has shown to be more versatile in 3D [99, 118]. Next to lamellipodium-based migration, lobopodial migration is observed in 3D for human fibroblasts [99]. During lobopodial migration, cells lack lamellipodia and polarization of Rac1 and Cdc42 activity, but instead display blunt cylindrical protrusions and lateral blebs. Although, to our knowledge, for 
ECs no lobopodia-based migration has been observed experimentally, it suggests that one cannot simply extrapolate information or models of 2D cell migration to 3D.

Secondly, beside the versatility in migration types, cell migration in 3D differs from 2D migration in the fact that proteolytic degradation of the ECM is required in $3 \mathrm{D}$. The cell needs to degrade and remodel its surrounding environment in order to be able to move forward, the extent of which depending on the ECM pore size, cell and migration type. Therefore, for modeling of cell migration and sprout formation in 3D a computational model of the ECM is required which should capture both the mechanical behavior of the ECM as well as molecular properties like degradation sites, adhesion sites and binding sites for pro-angiogenic signals. While previous CPM models have already modeled the ECM as obstacles for cells with which they can interact ( [5]), these interactions did not deal with mechanical interactions, and therefore such an approach has not been used before.

Finally, while the discussion on mechanochemical principles in angiogenesis models has focused so far on cell migration, this is not the only aspect that could benefit from and be extended with a mechanical description. The same could be true for cell division.

\subsection{Towards angiogenesis models that better capture molecular mechanisms}

Most of the models that we reviewed in Section 3. lack to a large extent the molecular interactions that we described in Appendix A and limit themselves to defining the primary signals (like e.g. VEGF, fibronectin, or MMP). For instance, mutual feedback and cross-talk have been evidenced between pro-angiogenic signals like VEGF, adhesion receptors like integrins (cell-matrix adhesion) and VE-cadherin (cell-cell adhesion) and matrix-degrading enzymes like MMPs, which together contribute to the modulation of cell-matrix and cell-cell interaction, and as such are important for EC migration and sprout lengthening. VEGF-induced Notch signaling controls cell-cell interaction (tip cell selection) and EC (migratory, proliferative and quiescent) phenotypes. The absence of these molecular interactions in most of the models that were reviewed here (in particular in models of Section 3.1.) should not be seen as a criticism on these models as in fact, most of them were created with another purpose in mind, and to study mechanisms at higher (length) scales. Nevertheless, given the fact that many of the current challenges in biology and medicine are being addressed at a molecular level, we feel it is important that some of the molecular players - which one obviously depend on the research question at hand - are explicitly accounted for in models of angiogenesis, so that their role for EC behavior can be incorporated. Detailed models of VEGF signaling, previously developed by Popel and co-workers (as reviewed in [121]) can be a starting basis. Examples of angiogenesis models that incorporate molecular interactions have been included in this review (see Section 3.2. and 3.3.), such as the work of Jain and Jackson who implemented chemotaxis, based on (polarity of) VEGFR-2 activation [63], or models that accounted for the role of Dll4 $[8,18,105]$. The work of Bentley and co-workers can serve as an example how a computational model of angiogenesis can help in shedding light on the role of certain molecular players, in particular Dll4-Notch signaling. Crucial for their models and the understanding that is gained from them, is the fact that cells are treated as dynamic entities that can change their shape in response to molecular signals that are activated by external stimuli. This enabled them among 
others to address the importance of filopodia formation for the dynamics of (tip) cell fate ( [8]) and to investigate mechanisms of cell-cell interaction that can potentially lead to cell rearrangements (shuffling) in a growing sprout ( [7]).

The incorporation of more mechanistic (cell migration) models that describe cell behavior as a consequence of molecular activity could also be useful to revisit simulation results on the importance of haptotaxis/haptokinesis and chemotaxis, as previously addressed by some of the reinforced random walk models $[2,86,96,100,119,124,129]$. This could for instance be done by including Rho GTPase activity and some of its upstream activators and downstream targets. As illustrated in Figure 2 Rho GTPase activity is modulated by transmembrane receptors involved in pro-angiogenic signaling (such as VEGFR-2 [32,58]) as well as adhesion receptors (such as integrins) [58]. As some of the ligands that are responsible for receptor activation are in fact the chemotactic (such as VEGF in the case of VEGFR-2 activation) or haptotactic cues (such as ECM proteins in the case of integrin signaling-in) defined in the earlier mentioned more phenomenological models, it means that chemotactic and haptotactic phenomena converge (at least partly) to similar molecular mechanisms and that there exists important cross-talk between the underlying signals. Besides, both pro-angiogenic signals (through VEGFR2 activation) and mechanical signals (through focal adhesions) have shown to regulate the Rho GTPase distribution in the cell (see [58]), demonstrating again the importance of having synergy between molecular signaling and mechanics in a model of angiogenesis.

\subsection{Towards multiscale mechanochemical models of angiogenesis}

In the previous sections we have discussed the importance of molecular and mechanical mechanisms for angiogenesis and to what extent current models of angiogenesis have incorporated these mechanisms. Here we want to make a concrete suggestion of how current models of angiogenesis and cell migration could be combined to come up with a multiscale mechanochemical model of angiogenesis. Ideally, such a model should capture and integrate various facets of angiogenesis (as is illustrated in Figure 2. One could see VEGFR2-activation and Rho GTPase dynamics as a central module in such a model, since they form the link between the biological and mechanical aspects of angiogenesis. The model of Marée et al. describes the arrangement of the Rho GTPases in a polarized cell (see Section 5.1.) and could serve as a starting point [82]. A second module is then required that facilitates polarization of the cell due to activation of VEGFR2 on the cell membrane by soluble and ECM-bound VEGF molecules. Models have already been developed that describe the dynamics of VEGF in angiogenesis (see [121] for a review). Based on current knowledge of the downstream effect of VEGFR2 on Rho GTPases [15,73] a link should be made between VEGF dynamics and cell mechanics in angiogenesis. Furthermore, the effect of VEGFR2 activation on cell-cell competition via Notch signaling $[8,10,64]$ and via VE-cadherin based cell repositioning [7], and on ECM degradation through secreted and membrane bound MMPs [66] could be incorporated, of course dependent on the purpose of the model.

The molecular modules proposed so far that couple pro-angiogenic signaling to Rho GTPase rearrangement should then be coupled to a module that incorporates the molecular mechanical principles of cell mechanics. Such a model should be able to describe protrusive forces produced 
by the lamellipodium by a combination of actin polymerization, branching, capping, coupling to the ECM via nascent adhesions and contraction by NM Myosin II (see e.g. [136, 137]). Besides, a discrete representation and continuous turnover of focal adhesions is required, where actomyosin is likely to play a role in adhesion disconnection at the rear of the cell by applying traction forces (as reviewed by $[24,42,137])$. The model developed by Shao et al., in which an actin network represented as a viscous fluid deforms as a result of actomyosin contraction, actin filament polymerization and discrete adhesions, could serve to couple Rho GTPase rearrangements to cell mechanics. However, it has to be kept in mind that this model is a 2D model and would require extension to become 3D. This model could be combined with adhesion maturation as described in the model of Stéphanou et al. [122]. Besides, a mechanical cell model as used by Herant and Dembo or Odenthal et al. could be used to secure a mechanically sound model $[49,50,93]$. Modeling of cell proliferation in a mechanically correct way, which is also required for angiogenic sprout elongation, is another challenge which will not be discussed further in this review paper.

So far, this model would describe the effects of pro-angiogenic signals on cell mechanical (adhesional, cytoskeletal) behavior, describing mechanochemical interactions in only one direction, i.e. going from chemical signals to mechanical responses. It is well known that these interactions are in fact bidirectional, because mechanical forces can modulate chemical activity of molecules. This is possible because forces that are transduced through e.g. focal adhesions and the cytoskeleton can deform molecules that are bound to or that are part of these force-transducing structures, in this way inducing conformational changes and therefore changes in molecular activity $[23,54,60,140,148]$. Such a feedback emphasizes the need for a tight coupling between molecular signaling and mechanics in computational models of angiogenesis. Since the force generation by the lamellipodium and stress fibers and the feedback between mechanics and molecular signaling occur at the molecular level, models must be refined enough at both the temporal and spatial level to capture these mechanisms. At the same time, such a model should be able to describe the behavior of multiple cells at the time scale of hours or days. Therefore, as was discussed before by Qutub et al., multiscale models are inevitable for modeling of angiogenesis [104].

\subsection{Link between computational models and experiments}

In Section 6.3. we have suggested to couple current computational models of angiogenesis and cell migration to come to multiscale mechanochemical models of (sprouting) angiogenesis. The coupling can be expected to be challenging from a computational (implementation) point of view, as well as concerning information management. The computational implementation of molecular mechanisms and cell-matrix mechanical interactions is likely to require some hybrid approach. Information management can be challenging, because of the vast amount of information available in the literature on molecular and mechanochemical feedback mechanisms, making it far from straightforward to make the right choices on the kind of mechanisms and interactions that should be implemented. Clearly, having a specific research question related to a specific experimental model or condition (either in vivo or in vitro) will help in making these choices. This also brings us to a final, more general suggestion that is key to enhancing the impact of computational models, and which is the relation between computational models and experiments. In order to make 
sure computational models can increase our understanding of angiogenesis, computational models should be calibrated and validated. Typically this requires an iterative approach between models and experiments as was already stated before (see $[9,98])$, where after a first calibration phase, hypotheses generated by the computational models are verified experimentally, which in turn may lead to more or refined research questions, model updates and improvements etc. This is definitely also true for ensuring 'mechanically sound' models of angiogenesis, which would require the acquisition of data on cell and matrix mechanical performance, which has become possible due to the emergence of advanced cell mechanical techniques, such as atomic force microscopy, optical tweezers, magnetic twisting cytometry and traction force microscopy [62]. Not surprisingly, it means that in order to improve computational models of angiogenesis and to generate novel understanding by means of model simulations, an interdisciplinary approach is needed that combines computational and experimental methods.

\section{Appendix}

\section{A Molecular signals during sprouting angiogenesis}

A. Pro-angiogenic signaling: Growth factors from the VEGF family of growth factors diffuse and bind to cell surface receptor tyrosine kinases called VEGFR. More specifically, binding of VEGF-A to VEGFR-2 plays a central role in sprouting angiogenesis [20]. Binding of these receptors is amplified by co-receptors called neuropilins [123]. VEGF can also be bound to the matrix (mVEGF) [57] or sequestered by soluble VEGFR-1 (sVEGFR-1) in regions next to an elongating sprout [43]. The VEGF ligand-receptor interaction induces dimerization of the receptor, and activates multiple well characterized signaling cascades such as the MAPK/ERK pathway (RasRaf-MEK-ERK), the Rho-kinase/myosin light chain kinase pathway (Rho/MLCK) and the phosphatidylinositol 3-Kinase/AKT pathway (PI3K/Akt) which regulate EC migration, proliferation and cell survival [11]. VEGF signaling also activates Notch signaling (see D. Cell-cell signaling) to laterally inhibit neighboring cells to become a tip cell, in this way regulating the initial and ongoing competition for the tip cell position in the sprout [64].

B. Cell-matrix signaling: At the interface between cell and ECM, transmembrane integrins are bound with their extracellular tail to the matrix and with their intracellular tail to the cytoskeleton in a protein complex including vinculin, talin, FAK and $\alpha$-actinin. Integrin signaling activates cytosolic proteins and signaling cascades such as the previously mentioned MAPK/ERK, Rho/MLCK and PI3K/Akt pathways [111,120], regulating in this way cytoskeletal rearrangements, cell polarity and proliferation. Integrin signaling is a classic example of bidirectional signaling. Signaling-out occurs when an intracellular activator, such as talin, interacts with the cytosolic subunit leading to a more accessible conformation with increased affinity for extracellular ligands. Signaling-in occurs in response to ligand interactions. This ligand binding often contributes to integrin clustering because many ligands are multivalent, possessing multiple binding sites [113].

C. Signals involved in cytoskeletal rearrangements: Downstream of VEGFR and integrins (and 
many other receptors), Rho GTPases are activated [32,58], of which RhoA, Rac1 and Cdc42 have been most extensively reviewed $[48,117]$, and have been linked to angiogenesis $[15,131]$ and vascular permeability [143]. They play a central role in the crosstalk between many signaling pathways leading to cytoskeletal rearrangements (For details on cytoskeletal dynamics, see Appendix B). Nascent focal complexes are formed in response to Rac1 signaling, while the maturation of focal complexes into focal adhesions occurs in response to RhoA signaling [25]. Rac1 signaling is further associated with lamellipodium formation, RhoA with stress fiber formation, and Cdc42 with filopodium formation [32].

D. Cell-cell signaling: Endothelial tips cells show higher expression of delta-like ligand 4 (Dll4) to interact with Notch receptors expressed on the adjacent stalk cells. This signaling results in upregulated Notch activity and downregulation of VEGFR-2 expression in the stalk cells, which renders them less responsive to VEGF [39]. This signaling also leads to an upregulation of soluble VEGFR-1 which sequesters VEGF [43]. ECs aligning blood vessels express a panoply of junctional proteins to form a tight layer, to control vascular permeability and to assure cell-cell communication. Typical junction proteins between ECs involved in angiogenesis are vascular endothelial (VE)-cadherins in association with $\beta$-catenins, and platelet EC adhesion molecule-1 (PECAM-1) [115]. Long-range communication in vessels is accomplished by cell-cell communication through connexins [20] forming gap junctions which enable rapid transport of $\mathrm{Ca}^{2+}$ and inositol trisphosphate (IP3) between ECs [95]. For short-range communication ECs have tight junctions consisting of, among others, claudins, occludins and junctional adhesion molecules (JAMs), to maintain barriers and adherens junctions to establish cell-cell adhesion, cytoskeleton remodeling and intracellular signaling [34].

E. Signals involved in matrix remodeling: Both VEGF signaling and integrin signaling [147] induce ECs to secrete matrix degrading enzymes such as matrix metalloproteinases (MMPs), allowing the sprout to invade into the matrix. While most MMPs are secreted, some are membrane associated (membrane-type, MT-MMPs). Matrix degradation exposes cryptic sites with growth factors, and thus activates VEGF signaling in a positive feedback loop. Some MMPs cleave among others pro-inflammatory cytokines, which also results in a pro-angiogenic signal. Proteases also negatively control angiogenesis, as some proteolytic fragments of the ECM possess anti-angiogenic properties, such as fibulin (basement membrane-derived) and endostatin (collagen XVIII-derived) [91]. Extensive reviews have been written about enzymes linked to endothelial sprouting and angiogenesis $[109,132]$ and ECM remodeling $[30,45]$.

\section{B The cytoskeleton, actin polymerization and protrusive and traction forces}

The central hub for cellular movement is the cytoskeleton. The cytoskeleton is a biopolymer network consisting of three major components: actin filaments, microtubules and intermediate filaments [102]. The organization of the cytoskeleton is modified in response to chemical cues (receptor activation through ligand binding), such as VEGF, and mechanical cues through signaling cascades that for both type of cues involve among others the Rho GTPases [15, 58, 103, 117, 131] 
(See also Appendix A). For active cell migration, actin is the most important component since it generates membrane protrusions and can bind to myosin to form contractile stress fibers. Microtubules and intermediate filaments are components that among others generate the stiffness and stability of the cell $[13,51]$. Globular $(\mathrm{G})$-actin proteins polymerize to form actin filaments (Factin) of approximately $7 \mathrm{~nm}$ diameter [69]. Actin networks can be found in the cell cortex, in long parallel bundles in the filopodia, highly branched in lamellipodia and in tight coupling with non-muscle (NM) Myosin II in stress fibers. In proliferating cells actin also forms a contractile ring to separate the two daughter cells during cytokinesis. Microtubules are rigid $25 \mathrm{~nm}$ hollow rods composed of a single type of globular proteins called tubulin [139]. They have functions in cell movement, intracellular transport and the separation of chromosomes during mitosis. Intermediate filaments such as vimentin typically form $10 \mathrm{~nm}$ filaments [44]. The protein composition of the subunits is not as conserved as for actin and tubulin. These subunits form a broad class of proteins and are encoded by more than 70 different genes in vertebrates and invertebrates [44]. Crosstalk between actin, microtubules and intermediate filaments emerges from recent research as reviewed by Bayless and Johnson [6]. In this review the focus will be mainly on the actin component of the cytoskeleton.

The dynamic actin network is continuously remodeled by polymerization and depolymerization, involving some commonly accepted mechanisms (see also Figure 1) which are described extensively in literature [25, 102]. G-actin monomers with ATP are added in a high rate to filamentous (F)-actin polymers at the barbed end side near the membrane and at a slower rate at the pointed end of the polymer. F-actin depolymerizes due to the hydrolysis of ATP into ADP, which reduces the affinities of the monomers to stay bound. Many chaperone proteins are involved in actin dynamics. Capping proteins bind to the growing ends and terminate elongation. The Arp2/3 protein complex and formins such as mDia1 and mDia2 [138] nucleate actin polymerization. Formins not only promote nucleation, but also promote barbed end elongation [36]. The Arp2/3 complex promotes actin filament branching. Cofilin severs F-actin, which creates free barbed ends where actin polymerization can be nucleated, and contributes in this way to the control of the temporal and spatial extent of actin dynamics [14]. Profilin catalyzes the exchange of ADP for ATP of the actin monomers, creating a recycled pool of ADP-actin monomers that can be added to the growing end.

Cells that migrate on a flat substrate protrude their leading edge and retract their rear in a coordinated fashion. Membrane protrusions occur by actin polymerization of the lamellipodium at the membrane site. If the lamellipodium is not constrained to the ECM via integrins, the membrane will push the growing actin polymers backwards, creating a retrograde flow [25]. This retrograde flow can be fast and independent of NM myosin II in the lamellipodium, but also slow and enhanced by contractions of NM myosin II in stress fibers in the lamella [137]. If the actin polymers are constrained to the substrate via integrins, the polymers cannot be pushed back by the membrane, resulting in the creation of a protrusive force and membrane protrusion [25,137].

ECs exert traction forces that can realign the matrix [72]. These traction forces are generated by stress fibers composed of actin bundles and bipolar NM myosin II filaments (see also Figure 2). Depending on their shape and composition stress fibers can be classified as ventral stress fibers, dorsal stress fibers and transverse arcs, as reviewed elsewhere [56,89]. These contractile structures are often anchored to focal adhesions, where traction forces can be transmitted to the substrate. 
The integrin receptors organize a cytoskeletal signaling complex within the focal adhesion to preferentially focus mechanical forces on this site [59]. 


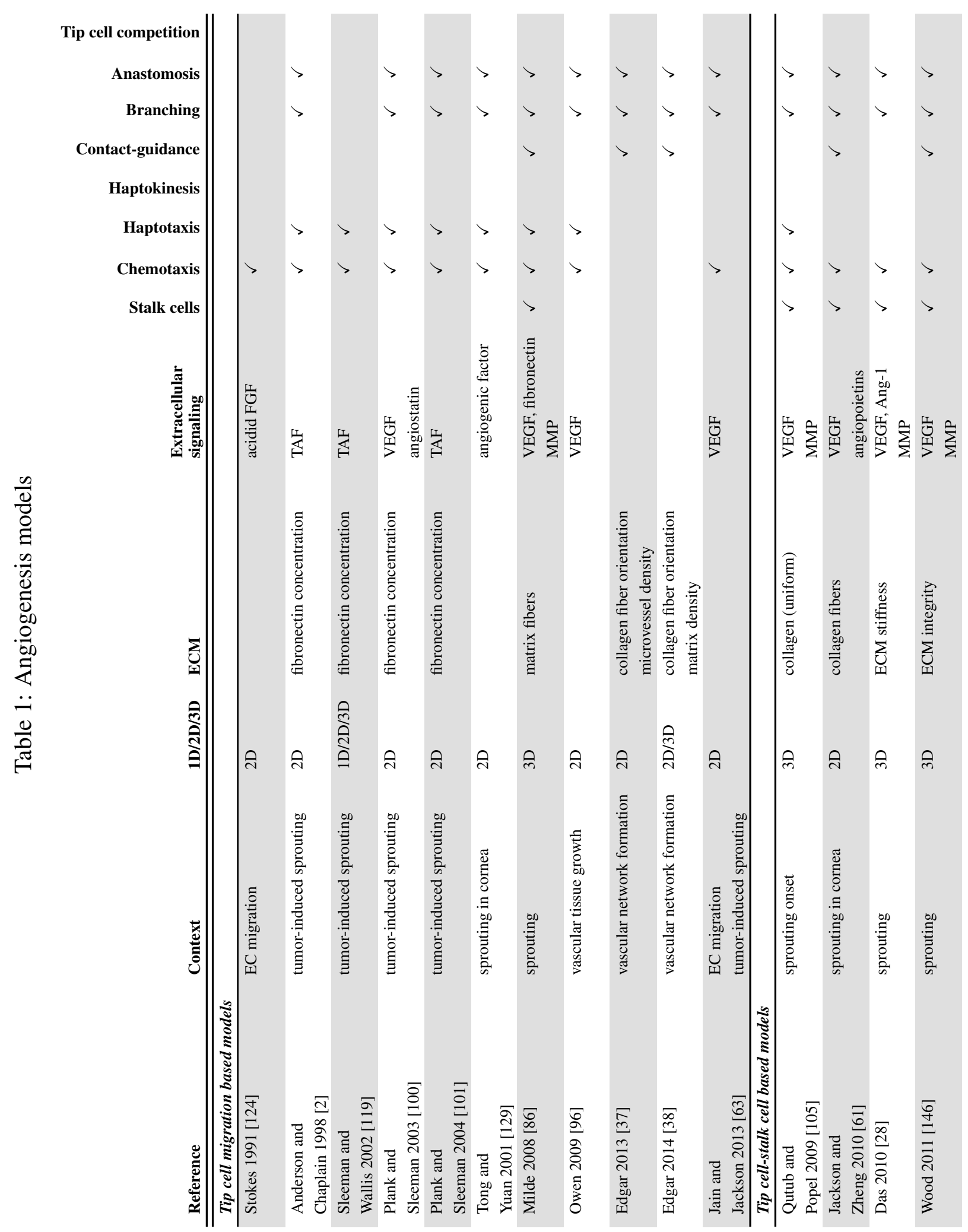




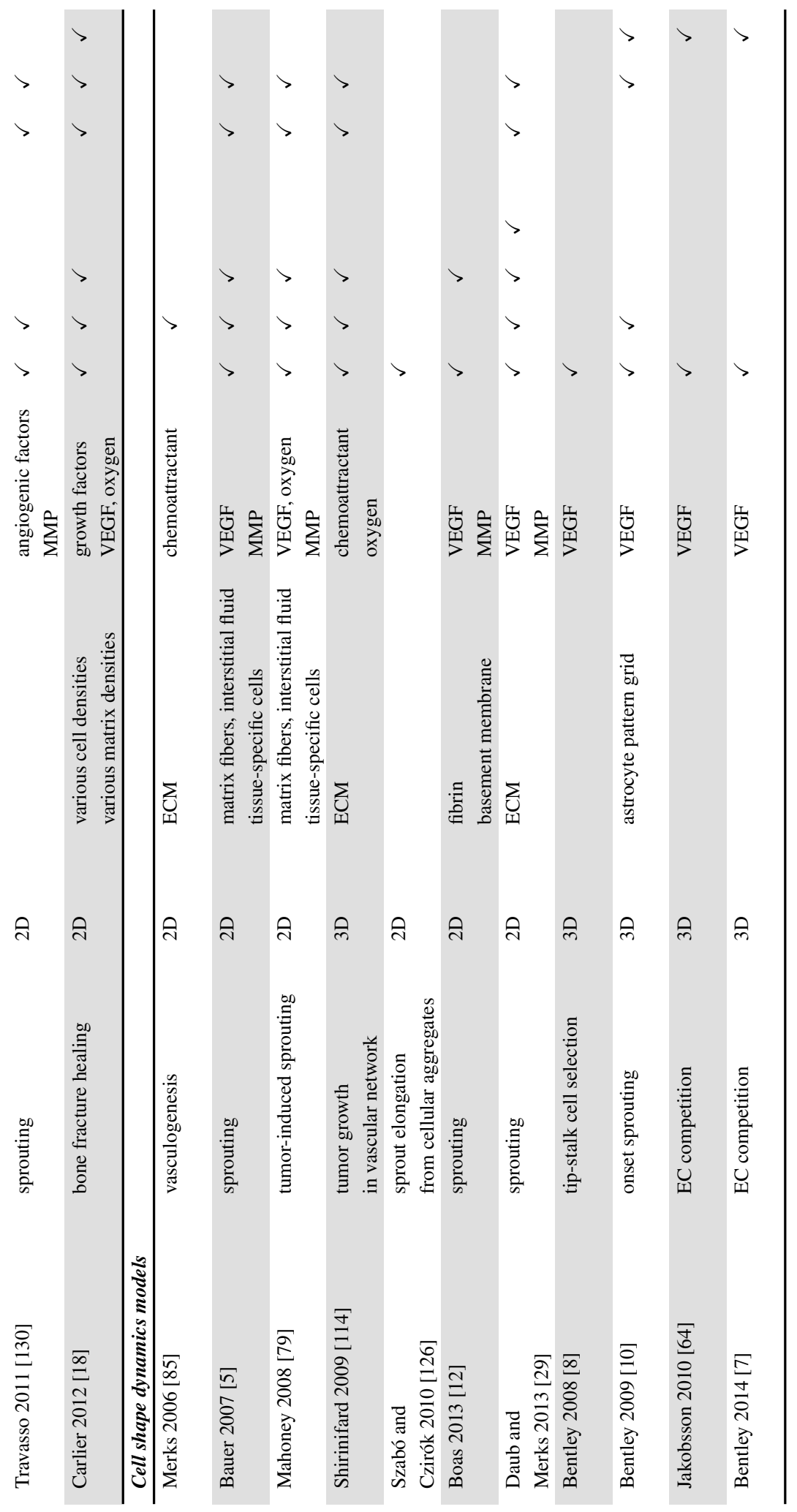




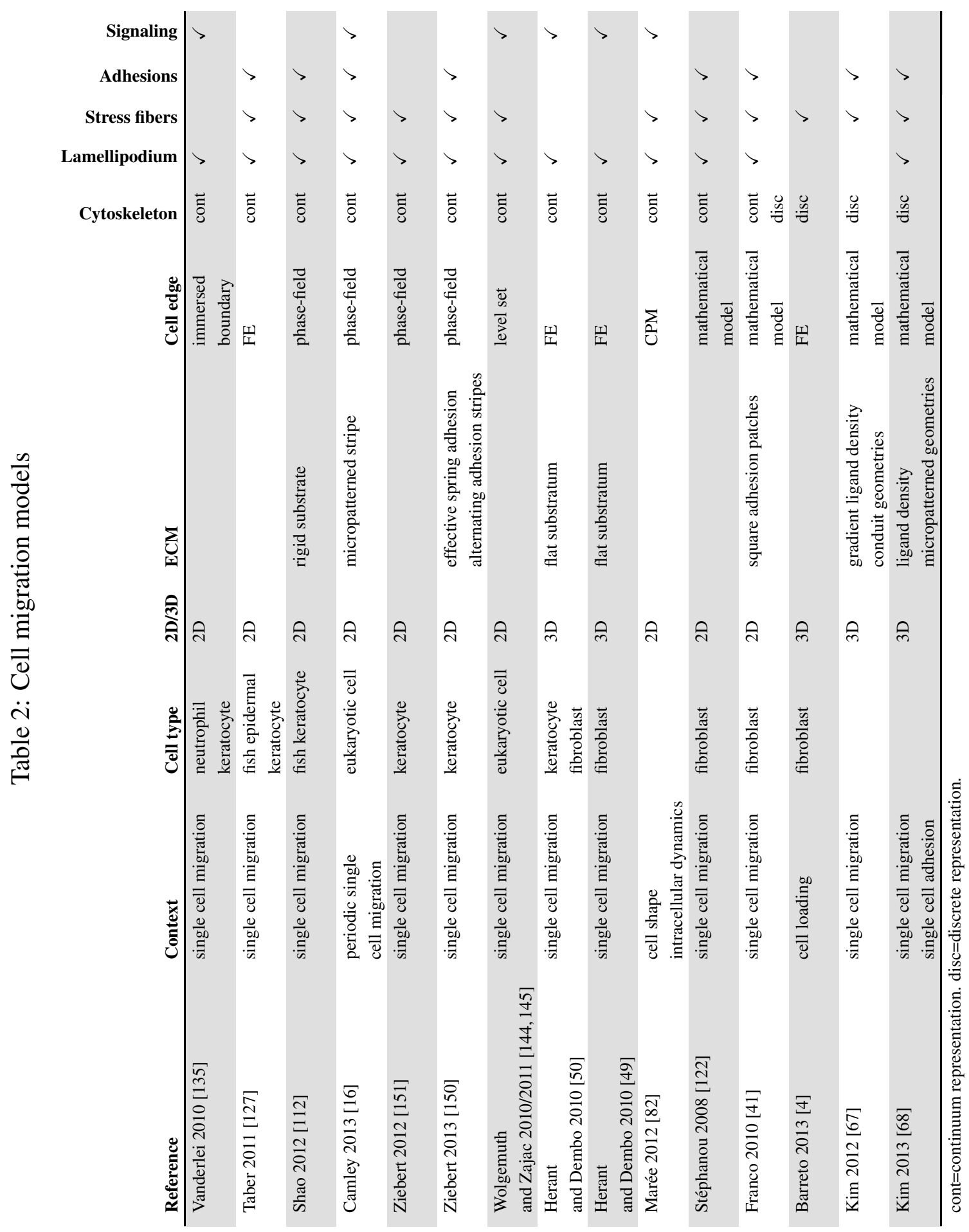




\section{Acknowledgements}

The research leading to these results has received funding from the European Research Council under the European Union's Seventh Framework Programme (FP7/2007-2013)/ ERC Grant Agreement $\mathrm{n}^{\circ}$ 308223) and from the Research Fund - Flanders (FWO-Vlaanderen, grant number G.0821.13). Tommy Heck is a PhD fellow of FWO-Vlaanderen.

\section{References}

[1] W. Alt, R. T. Tranquillo Basic morphogenetic system modeling shape changes of migrating cells: how to explain fluctuating lamellipodial dynamics. J. Biol. Syst. 3(4) (1995), 905916.

[2] A. R. Anderson, M. A. Chaplain Continuous and discrete mathematical models of tumorinduced angiogenesis. Bull. Math. Biol. 60(5) (1998), 857-900.

[3] S. Arima, K. Nishiyama, T. Ko, Y. Arima, Y. Hakozaki, K. Sugihara, H. Koseki, Y. Uchijima, Y. Kurihara, H. Kurihara Angiogenic morphogenesis driven by dynamic and heterogeneous collective endothelial cell movement. Development 138(21) (2011), 4763-4776.

[4] S. Barreto, C. H. Clausen, C. M. Perrault, D. a. Fletcher, D. Lacroix A multi-structural single cell model of force-induced interactions of cytoskeletal components. Biomaterials 34(26) (2013), 6119-6126.

[5] A. L. Bauer, T. L. Jackson, Y. Jiang A cell-based model exhibiting branching and anastomosis during tumor-induced angiogenesis. Biophys. J. 92(9) (2007), 3105-3121.

[6] K. J. Bayless, G. A. Johnson Role of the cytoskeleton in formation and maintenance of angiogenic sprouts. J. Vasc. Res. 48(5) (2011), 369-385.

[7] K. Bentley, C. A. Franco, A. Philippides, R. Blanco, M. Dierkes, V. Gebala, F. Stanchi, M. Jones, I. M. Aspalter, G. Cagna, S. Weström, L. Claesson-welsh, D. Vestweber, H. Gerhardt The role of differential VE-cadherin dynamics in cell rearrangement during angiogenesis. Nat. Cell Biol. 16 (2014), 309-321.

[8] K. Bentley, H. Gerhardt, P. A. Bates Agent-based simulation of notch-mediated tip cell selection in angiogenic sprout initialisation. J. Theor. Biol. 250(1) (2008), 25-36.

[9] K. Bentley, M. Jones, B. Cruys Predicting the future: towards symbiotic computational and experimental angiogenesis research. Exp. Cell Res. 319(9) (2013), 1240-1246.

[10] K. Bentley, G. Mariggi, H. Gerhardt, P. A. Bates Tipping the balance: robustness of tip cell selection, migration and fusion in angiogenesis. PLoS Comput. Biol. 5(10) (2009), e1000549. 
[11] R. Blanco, H. Gerhardt VEGF and Notch in tip and stalk cell selection. Cold Spring Harb. Perspect. Med. 3(1) (2013), a006569.

[12] S. Boas, M. Palm, P. Koolwijk, R. Merks Computational modeling of angiogenesis: towards a multi-scale understanding of cellcell and cellmatrix interactions in $\mathrm{C}$. A. Reinhart-King, editor, Mech. Chem. Signal. Angiogenes. SE - 8 vol. 12 of Studies in Mechanobiology, Tissue Engineering and Biomaterials Springer Berlin Heidelberg ISBN 978-3-642-30855-0 (2013) 161-183.

[13] C. P. Brangwynne, F. C. MacKintosh, S. Kumar, N. A. Geisse, J. Talbot, L. Mahadevan, K. K. Parker, D. E. Ingber, D. A. Weitz Microtubules can bear enhanced compressive loads in living cells because of lateral reinforcement. J. Cell Biol. 173(5) (2006), 733-741.

[14] J. J. Bravo-Cordero, M. A. O. Magalhaes, R. J. Eddy, L. Hodgson, J. Condeelis Functions of cofilin in cell locomotion and invasion. Nat. Rev. Mol. Cell Biol. 14(7) (2013), 405-415.

[15] B. A. Bryan, P. A. D’Amore What tangled webs they weave: Rho-GTPase control of angiogenesis. Cell. Mol. Life Sci. 64(16) (2007), 2053-2065.

[16] B. A. Camley, Y. Zhao, B. Li, H. Levine, W.-J. Rappel Periodic migration in a physical model of cells on micropatterns. Phys. Rev. Lett. 111(15) (2013), 158102.

[17] L. Cardamone, A. Laio, V. Torre, R. Shahapure, A. DeSimone Cytoskeletal actin networks in motile cells are critically self-organized systems synchronized by mechanical interactions. Proc. Natl. Acad. Sci. 108(34) (2011), 13978-13983.

[18] A. Carlier, L. Geris, K. Bentley, G. Carmeliet, P. Carmeliet, H. Van Oosterwyck MOSAIC: a multiscale model of osteogenesis and sprouting angiogenesis with lateral inhibition of endothelial cells. PLoS Comput. Biol. 8(10) (2012), e1002724.

[19] P. Carmeliet, F. De Smet, S. Loges, M. Mazzone Branching morphogenesis and antiangiogenesis candidates: tip cells lead the way. Nat. Rev. Clin. Oncol. 6(6) (2009), 315-326.

[20] P. Carmeliet, R. K. Jain Molecular mechanisms and clinical applications of angiogenesis. Nature 473(7347) (2011), 298-307.

[21] J. C. Chappell, D. M. Wiley, V. L. Bautch Regulation of blood vessel sprouting. Semin. Cell Dev. Biol. 22(9) (2011), 1005-1011.

[22] S. Checa, P. J. Prendergast A mechanobiological model for tissue differentiation that includes angiogenesis: a lattice-based modeling approach. Ann. Biomed. Eng. 37(1) (2009), 129-145.

[23] C. S. Chen Mechanotransduction - a field pulling together? J. Cell Sci. 121(Pt 20) (2008), 3285-3292. 
[24] Q. Chi, T. Yin, H. Gregersen, X. Deng, Y. Fan, J. Zhao, D. Liao, G. Wang Rear actomyosin contractility-driven directional cell migration in three- dimensional matrices: a mechanochemical coupling mechanism. J. R. Soc. Interface 11(95) (2014), 20131072.

[25] C. L. E. Clainche, M.-F. Carlier Regulation of actin assembly associated with protrusion and adhesion in cell migration. Physiol. Rev. 88(2) (2008), 489-513.

[26] V. L. Cross, Y. Zheng, N. Won Choi, S. S. Verbridge, B. a. Sutermaster, L. J. Bonassar, C. Fischbach, A. D. Stroock Dense type I collagen matrices that support cellular remodeling and microfabrication for studies of tumor angiogenesis and vasculogenesis in vitro. Biomaterials 31(33) (2010), 8596-8607.

[27] G. Danuser, J. Allard, A. Mogilner Mathematical modeling of eukaryotic cell migration: insights beyond experiments. Annu. Rev. Cell Dev. Biol. 29 (2013), 501-528.

[28] A. Das, D. Lauffenburger, H. Asada, R. D. Kamm A hybrid continuumdiscrete modelling approach to predict and control angiogenesis: analysis of combinatorial growth factor and matrix effects on vessel-sprouting. Philos. Trans. R. Soc. A 368 (2010), 2937-2960.

[29] J. T. Daub, R. M. H. Merks A cell-based model of extracellular-matrix-guided endothelial cell migration during angiogenesis. Bull. Math. Biol. 75(8) (2013), 1377-1399.

[30] G. E. Davis, D. R. Senger Endothelial extracellular matrix: biosynthesis, remodeling, and functions during vascular morphogenesis and neovessel stabilization. Circ. Res. 97(11) (2005), 1093-1107.

[31] A. T. Dawes, L. Edelstein-Keshet Phosphoinositides and Rho proteins spatially regulate actin polymerization to initiate and maintain directed movement in a one-dimensional model of a motile cell. Biophys. J. 92(3) (2007), 744-768.

[32] F. De Smet, I. Segura, K. De Bock, P. J. Hohensinner, P. Carmeliet Mechanisms of vessel branching: filopodia on endothelial tip cells lead the way. Arterioscler. Thromb. Vasc. Biol. 29(5) (2009), 639-649.

[33] E. Dejana Endothelial cell-cell junctions: happy together. Nat. Rev. Mol. Cell Biol. 5(4) (2004), 261-270.

[34] E. Dejana, E. Tournier-Lasserve, B. M. Weinstein The control of vascular integrity by endothelial cell junctions: molecular basis and pathological implications. Dev. Cell 16(2) (2009), 209-221.

[35] J. A. Ditlev, N. M. Vacanti, I. L. Novak, L. M. Loew An open model of actin dendritic nucleation. Biophys. J. 96(9) (2009), 3529-3542.

[36] R. Dominguez Structural insights into de novo actin polymerization. Curr. Opin. Struct. Biol. 20(2) (2010), 217-225. 
[37] L. T. Edgar, S. C. Sibole, C. J. Underwood, J. E. Guilkey, J. A. Weiss A computational model of in vitro angiogenesis based on extracellular matrix fibre orientation. Comput. Methods Biomech. Biomed. Engin. 16(7) (2013), 790-801.

[38] L. T. Edgar, C. J. Underwood, J. E. Guilkey, J. B. Hoying, J. A. Weiss Extracellular matrix density regulates the rate of neovessel growth and branching in sprouting angiogenesis. PLoS One 9(1) (2014), e85178.

[39] H. M. Eilken, R. H. Adams Dynamics of endothelial cell behavior in sprouting angiogenesis. Curr. Opin. Cell Biol. 22(5) (2010), 617-625.

[40] R. S. Fischer, M. Gardel, X. Ma, R. S. Adelstein, C. M. Waterman Local cortical tension by myosin II guides 3D endothelial cell branching. Curr. Biol. 19(3) (2009), 260-265.

[41] C. Franco, T. Tzvetkova-Chevolleau, A. Stéphanou On the Influence of Discrete Adhesive Patterns for Cell Shape and Motility: A Computational Approach. Math. Model. Nat. Phenom. 5(1) (2010), 56-83.

[42] M. L. Gardel, I. C. Schneider, Y. Aratyn-Schaus, C. M. Waterman Mechanical integration of actin and adhesion dynamics in cell migration. Annu. Rev. Cell Dev. Biol. 26 (2010), 315-333.

[43] I. Geudens, H. Gerhardt Coordinating cell behaviour during blood vessel formation. Development 138(21) (2011), 4569-4583.

[44] R. D. Goldman, M. M. Cleland, S. N. P. Murthy, S. Mahammad, E. R. Kuczmarski Inroads into the structure and function of intermediate filament networks. J. Struct. Biol. 177(1) (2012), 14-23.

[45] S. J. Grainger, A. J. Putnam Mechanical and chemical signaling in angiogenesis in C. A. Reinhart-King, editor, Mech. Chem. Signal. Angiogenes. vol. 12 of Studies in Mechanobiology, Tissue Engineering and Biomaterials Springer Berlin Heidelberg, Berlin, Heidelberg ISBN 978-3-642-30855-0 (2013) 185-209.

[46] F. Graner, J. A. Glazier Simulation of biological cell sorting using a two-dimensional extended potts model. Phys. Rev. Lett. 69(13) (1992), 2013-2016.

[47] H. P. Grimm, A. B. Verkhovsky, A. Mogilner, J.-J. Meister Analysis of actin dynamics at the leading edge of crawling cells : implications for the shape of keratocyte lamellipodia. Eur. Biophys. J. 32 (2003), 563-577.

[48] S. J. Heasman, A. J. Ridley Mammalian Rho GTPases: new insights into their functions from in vivo studies. Nat. Rev. Mol. Cell Biol. 9(9) (2008), 690-701.

[49] M. Herant, M. Dembo Cytopede: a three-dimensional tool for modeling cell motility on a flat surface. J. Comput. Biol. 17(12) (2010), 1639-1677. 
[50] M. Herant, M. Dembo Form and function in cell motility: from fibroblasts to keratocytes. Biophys. J. 98(8) (2010), 1408-1417.

[51] H. Herrmann, S. V. Strelkov, P. Burkhard, U. Aebi Intermediate filaments : primary determinants of cell architecture and plasticity. J. Clin. Invest. 119(7) (2009), 1772-1783.

[52] C. Hetheridge, A. N. Scott, R. K. Swain, J. W. Copeland, H. N. Higgs, R. Bicknell, H. Mellor The formin FMNL3 is a cytoskeletal regulator of angiogenesis. J. Cell Sci. 125(Pt 6) (2012), $1420-1428$.

[53] A. C. Hielscher, S. Gerecht Engineering approaches for investigating tumor angiogenesis: exploiting the role of the extracellular matrix. Cancer Res. 72(23) (2012), 6089-6096.

[54] B. D. Hoffman, C. Grashoff, M. A. Schwartz Dynamic molecular processes mediate cellular mechanotransduction. Nature 475(7356) (2011), 316-323.

[55] W. R. Holmes, L. Edelstein-Keshet A comparison of computational models for eukaryotic cell shape and motility. PLoS Comput. Biol. 8(12) (2012), e1002793.

[56] P. Hotulainen, P. Lappalainen Stress fibers are generated by two distinct actin assembly mechanisms in motile cells. J. Cell Biol. 173(3) (2006), 383-394.

[57] H. Hutchings, N. Ortega, J. Plouët Extracellular matrix-bound vascular endothelial growth factor promotes endothelial cell adhesion, migration, and survival through integrin ligation. FASEB J. 17(11) (2003), 1520-1522.

[58] S. Huveneers, E. H. J. Danen Adhesion signaling - crosstalk between integrins, Src and Rho. J. Cell Sci. 122(Pt 8) (2009), 1059-1069.

[59] D. E. Ingber Mechanical signaling and the cellular response to extracellular matrix in angiogenesis and cardiovascular physiology. Circ. Res. 91(10) (2002), 877-887.

[60] D. E. Ingber Tensegrity-based mechanosensing from macro to micro. Prog. Biophys. Mol. Biol. 97(2-3) (2008), 163-179.

[61] T. Jackson, X. Zheng A cell-based model of endothelial cell migration, proliferation and maturation during corneal angiogenesis. Bull. Math. Biol. 72(4) (2010), 830-868.

[62] C. R. Jacobs, H. Huang, R. Y. Kwon Introduction to cell mechanics and mechanobiology Garland Science (2012).

[63] H. V. Jain, T. L. Jackson A hybrid model of the role of VEGF binding in endothelial cell migration and capillary formation. Front. Oncol. 3 (2013), 102.

[64] L. Jakobsson, C. A. Franco, K. Bentley, R. T. Collins, B. Ponsioen, I. M. Aspalter, I. Rosewell, M. Busse, G. Thurston, A. Medvinsky, S. Schulte-Merker, H. Gerhardt Endothelial cells dynamically compete for the tip cell position during angiogenic sprouting. Nat. Cell Biol. 12(10) (2010), 943-953. 
[65] A. Jilkine, A. F. M. Marée, L. Edelstein-Keshet Mathematical model for spatial segregation of the Rho-family GTPases based on inhibitory crosstalk. Bull. Math. Biol. 69(6) (2007), 1943-1978.

[66] E. D. Karagiannis, A. S. Popel Distinct modes of collagen type I proteolysis by matrix metalloproteinase (MMP) 2 and membrane type I MMP during the migration of a tip endothelial cell: insights from a computational model. J. Theor. Biol. 238(1) (2006), 124-145.

[67] M.-C. Kim, C. Kim, L. Wood, D. Neal, R. D. Kamm, H. H. Asada Integrating focal adhesion dynamics, cytoskeleton remodeling, and actin motor activity for predicting cell migration on $3 D$ curved surfaces of the extracellular matrix. Integr. Biol. 4(11) (2012), 1386-1397.

[68] M.-C. Kim, D. M. Neal, R. D. Kamm, H. H. Asada Dynamic modeling of cell migration and spreading behaviors on fibronectin coated planar substrates and micropatterned geometries. PLoS Comput. Biol. 9(2) (2013), e1002926.

[69] A. Kishino, T. Yanagida Force measurements by micromanipulation of a single actin filament by glass needles. Nature 334(6177) (1988), 74-76.

[70] E. Kniazeva, A. J. Putnam Endothelial cell traction and ECM density influence both capillary morphogenesis and maintenance in 3-D. Am. J. Physiol. - Cell Physiol. 297(1) (2009), C179-C187.

[71] A. Köhn-Luque, W. de Back, J. Starruss, A. Mattiotti, A. Deutsch, J. M. Pérez-Pomares, M. a. Herrero Early embryonic vascular patterning by matrix-mediated paracrine signalling: a mathematical model study. PLoS One 6(9) (2011), e24175.

[72] T. Korff, H. G. Augustin Tensional forces in fibrillar extracellular matrices control directional capillary sprouting. J. Cell Sci. 112(Pt 19) (1999), 3249-3258.

[73] L. Lamalice, B. F. Le, J. Huot Endothelial cell migration during angiogenesis. Circ. Res. 100 (2007), 782-794.

[74] T. Lämmermann, M. Sixt Mechanical modes of 'amoeboid' cell migration. Curr. Opin. Cell Biol. 21(5) (2009), 636-644.

[75] P.-F. Lee, Y. Bai, R. L. Smith, K. J. Bayless, a. T. Yeh Angiogenic responses are enhanced in mechanically and microscopically characterized, microbial transglutaminase crosslinked collagen matrices with increased stiffness. Acta Biomater. 9(7) (2013), 7178-7190.

[76] C. A. Lemmon, L. H. Romer A predictive model of cell traction forces based on cell geometry. Biophys. journal2 99(9) (2010), L78-L80.

[77] G. Lemon, D. Howard, F. R. A. J. Rose, J. R. King Individual-based modelling of angiogenesis inside three-dimensional porous biomaterials. BioSystems 103(3) (2011), 372-83. 
[78] G. Liu, A. A. Qutub, P. Vempati, F. Mac Gabhann, A. S. Popel Module-based multiscale simulation of angiogenesis in skeletal muscle. Theor. Biol. Med. Model. 8 (2011), 6.

[79] A. W. Mahoney, B. G. Smith, N. S. Flann, G. J. Podgorski Discovering novel cancer therapies: A computational modeling and search approach. 2008 IEEE Symp. Comput. Intell. Bioinforma. Comput. Biol. (2008), 233-240.

[80] A. Mammoto, K. M. Connor, T. Mammoto, C. W. Yung, D. Huh, C. M. Aderman, G. Mostoslavsky, L. E. H. Smith, D. E. Ingber A mechanosensitive transcriptional mechanism that controls angiogenesis. Nature 457(7233) (2009), 1103-1108.

[81] D. Manoussaki, S. R. Lubkin, R. B. Vernon, J. D. Murray A mechanical model for the formation of vascular networks in vitro. Acta Biotheor. 44(3-4) (1996), 271-282.

[82] A. F. M. Marée, V. A. Grieneisen, L. Edelstein-Keshet How cells integrate complex stimuli: the effect of feedback from phosphoinositides and cell shape on cell polarization and motility. PLoS Comput. Biol. 8(3) (2012), e1002402.

[83] A. F. M. Marée, A. Jilkine, A. Dawes, V. A. Grieneisen, L. Edelstein-Keshet Polarization and movement of keratocytes: a multiscale modelling approach. Bull. Math. Biol. 68(5) (2006), 1169-1211.

[84] B. N. Mason, A. Starchenko, R. M. Williams, L. J. Bonassar, C. a. Reinhart-King Tuning three-dimensional collagen matrix stiffness independently of collagen concentration modulates endothelial cell behavior. Acta Biomater. 9(1) (2013), 4635-4644.

[85] R. M. H. Merks, S. V. Brodsky, M. S. Goligorksy, S. A. Newman, J. A. Glazier Cell elongation is key to in silico replication of in vitro vasculogenesis and subsequent remodeling. Dev. Biol. 289(1) (2006), 44-54.

[86] F. Milde, M. Bergdorf, P. Koumoutsakos A hybrid model for three-dimensional simulations of sprouting angiogenesis. Biophys. J. 95(7) (2008), 3146-3160.

[87] A. Mogilner, L. Edelstein-keshet Regulation of actin dynamics in rapidly moving cells : a quantitative analysis. Biophys. J. 83(3) (2002), 1237-1258.

[88] J. D. Murray Mathematical biology II: spatial models and biomedical applications Springer, Berlin 3 ed. (2003).

[89] P. Naumanen, P. Lappalainen, P. Hotulainen Mechanisms of actin stress fibre assembly. J. Microsc. 231(3) (2008), 446-454.

[90] S. Niland, J. A. Eble Integrin-mediated cell-matrix interaction in physiological and pathological blood vessel formation. J. Oncol. 2012 (2012), 125278.

[91] P. Nyberg, L. Xie, R. Kalluri Endogenous nhibitors of angiogenesis. Cancer Res. 65(10) (2005), 3967-3979. 
[92] G. Odell, G. Oster, P. Alberch, B. Burnside The mechanical basis of morphogenesis. Dev. Biol. 85(2) (1981), 446-462.

[93] T. Odenthal, B. Smeets, P. Van Liedekerke, E. Tijskens, H. Van Oosterwyck, H. Ramon Analysis of initial cell spreading using mechanistic contact formulations for a deformable cell model. PLoS Comput. Biol. 9(10) (2013), e1003267.

[94] B. G. F. Oster, J. D. Murray, A. K. Harris Mechanical aspects of mesenchymal morphogenesis. J. Embryol. Exp. Morphol. 78 (1983), 83-125.

[95] F. Otsuka, A. V. Finn, S. K. Yazdani, M. Nakano, F. D. Kolodgie, R. Virmani The importance of the endothelium in atherothrombosis and coronary stenting. Nat. Rev. Cardiol. 9(8) (2012), 439-453.

[96] M. R. Owen, T. Alarcón, P. K. Maini, H. M. Byrne Angiogenesis and vascular remodelling in normal and cancerous tissues. J. Math. Biol. 58(4-5) (2009), 689-721.

[97] V. Peiffer, A. Gerisch, D. Vandepitte, H. Van Oosterwyck, L. Geris A hybrid bioregulatory model of angiogenesis during bone fracture healing. Biomech. Model. Mechanobiol. 10(3) (2011), 383-395.

[98] S. M. Peirce, F. Mac Gabhann, V. L. Bautch Integration of experimental and computational approaches to sprouting angiogenesis. Curr. Opin. Hematol. 19(3) (2012), 184-191.

[99] R. J. Petrie, N. Gavara, R. S. Chadwick, K. M. Yamada Nonpolarized signaling reveals two distinct modes of 3D cell migration. J. Cell Biol. 197(3) (2012), 439-455.

[100] M. J. Plank, B. D. Sleeman A reinforced random walk model of tumour angiogenesis and anti-angiogenic strategies. Math. Med. Biol. 20(2) (2003), 135-181.

[101] M. J. Plank, B. D. Sleeman Lattice and non-lattice models of tumour angiogenesis. Bull. Math. Biol. 66(6) (2004), 1785-1819.

[102] T. D. Pollard The cytoskeleton, cellular motility and the reductionist agenda. Nature 422(6933) (2003), 741-745.

[103] P. P. Provenzano, P. J. Keely Mechanical signaling through the cytoskeleton regulates cell proliferation by coordinated focal adhesion and Rho GTPase signaling. J. Cell Sci. 124(Pt 8) (2011), 1195-1205.

[104] A. A. Qutub, F. Mac Gabhann, E. D. Karagiannis, P. Vempati, A. S. Popel Multiscale models of angiogenesis. IEEE Eng. Med. Biol. Mag. 28(2) (2009), 14-31.

[105] A. A. Qutub, A. S. Popel Elongation, proliferation \& migration differentiate endothelial cell phenotypes and determine capillary sprouting. BMC Syst. Biol. 3 (2009), 13. 
[106] R. Rangarajan, M. H. Zaman Modeling cell migration in 3D: Status and challenges. Cell Adh. Migr. 2(2) (2008), 106-109.

[107] B. Rubinstein, M. F. Fournier, K. Jacobson, A. B. Verkhovsky, A. Mogilner Actin-myosin viscoelastic flow in the keratocyte lamellipod. Biophys. J. 97(7) (2009), 1853-1863.

[108] B. Rubinstein, K. Jacobson, A. Mogilner Multiscale two-dimensional modeling of a motile simple-shaped cell. Multiscale Model. Simul. 3(2) (2005), 413-439.

[109] J. E. Rundhaug Matrix metalloproteinases and angiogenesis. J. Cell. Mol. Med. 9(2) (2005), $267-285$.

[110] D. R. Senger, C. A. Perruzzi, M. Streit, V. E. Koteliansky, A. R. de Fougerolles, M. Detmar The $\alpha 1 \beta 1$ and $\alpha 2 \beta 1$ Integrins Provide Critical Support for Vascular Endothelial Growth Factor Signaling, Endothelial Cell Migration, and Tumor Angiogenesis. Am. J. Pathol. 160(1) (2002), 195-204.

[111] G. Serini, L. Napione, F. Bussolino Integrins team up with tyrosine kinase receptors and plexins to control angiogenesis. Curr. Opin. Hematol. 15(3) (2008), 235-242.

[112] D. Shao, H. Levine, W.-J. Rappel Coupling actin flow, adhesion, and morphology in a computational cell motility model. Proc. Natl. Acad. Sci. U. S. A. 109(18) (2012), 6851-6856.

[113] S. J. Shattil, C. Kim, M. H. Ginsberg The final steps of integrin activation: the end game. Nat. Rev. Mol. Cell Biol. 11(4) (2010), 288-300.

[114] A. Shirinifard, J. S. Gens, B. L. Zaitlen, N. J. Popawski, M. Swat, J. a. Glazier 3D multi-cell simulation of tumor growth and angiogenesis. PLoS One 4(10) (2009), e7190.

[115] Y.-T. Shiu, J. A. Weiss, J. B. Hoying, M. N. Iwamoto, I. S. Joung, C. T. Quam The role of mechanical stresses in angiogenesis. Crit. Rev. Biomed. Eng. 33(5) (2005), 431-510.

[116] R. Silva, G. D’Amico, K. M. Hodivala-Dilke, L. E. Reynolds Integrins: the keys to unlocking angiogenesis. Arterioscler. Thromb. Vasc. Biol. 28(10) (2008), 1703-1713.

[117] S.-T. Sit, E. Manser Rho GTPases and their role in organizing the actin cytoskeleton. J. Cell Sci. 124(Pt 5) (2011), 679-683.

[118] M. Sixt Cell migration: Fibroblasts find a new way to get ahead. J. Cell Biol. 197(3) (2012), 347-349.

[119] B. Sleeman, I. Wallis Tumour induced angiogenesis as a reinforced random walk: Modelling capillary network formation without endothelial cell proliferation. Math. Comput. Model. 36(3) (2002), 339-358.

[120] P. R. Somanath, A. Ciocea, T. V. Byzova Integrin and growth factor receptor alliance in angiogenesis. Cell Biochem. Biophys. 53(2) (2009), 53-64. 
[121] M. O. Stefanini, A. A. Qutub, F. Mac Gabhann, A. S. Popel Computational models of VEGFassociated angiogenic processes in cancer. Math. Med. Biol. 29(1) (2012), 85-94.

[122] A. Stéphanou, E. Mylona, M. Chaplain, P. Tracqui A computational model of cell migration coupling the growth of focal adhesions with oscillatory cell protrusions. J. Theor. Biol. 253(4) (2008), 701-716.

[123] M. W. Stewart Vascular endothelial growth factor (VEGF) biochemistry and development of inhibitory drugs. Curr. Drug ther. 7(2) (2012), 80-89.

[124] C. L. Stokes, D. A. Lauffenburger, S. K. Williams Migration of individual microvessel endothelial cells: stochastic model and parameter measurement. J. Cell Sci. 99 ( Pt 2) (1991), 419-430.

[125] S.-c. Su, E. A. Mendoza, H.-i. Kwak, K. J. Bayless Molecular profile of endothelial invasion of three-dimensional collagen matrices : insights into angiogenic sprout induction in wound healing. Am. J. Physiol. 295(5) (2008), C1215-C1229.

[126] A. Szabó, A. Czirók The Role of Cell-Cell Adhesion in the Formation of Multicellular Sprouts. Math. Model. Nat. Phenom. 5(1) (2010), 106-122.

[127] Y. L. Taber, Y. Shi, L. Yang, P. Bayly A poroelastic model for cell crawling including mechanical coupling between cytoskeletal contraction and actin polymerization. J. Mech. Mater. Struct. 6(1-4) (2011), 569-589.

[128] M. Théry, M. Bornens Cell shape and cell division. Curr. Opin. Cell Biol. 18(6) (2006), 648-657.

[129] S. Tong, F. Yuan Numerical simulations of angiogenesis in the cornea. Microvasc. Res. 61(1) (2001), 14-27.

[130] R. D. M. Travasso, E. Corvera Poiré, M. Castro, J. C. Rodríguez-Manzaneque, J. C. Rodrguez-Manzaneque, A. Hernández-Machado Tumor angiogenesis and vascular patterning: a mathematical model. PLoS One 6(5) (2011), e19989.

[131] R. van der Meel, M. H. Symons, R. Kudernatsch, R. J. Kok, R. M. Schiffelers, G. Storm, W. M. Gallagher, A. T. Byrne The VEGF/Rho GTPase signalling pathway: a promising target for anti-angiogenic/anti-invasion therapy. Drug Discov. Today 16(5-6) (2011), 219228.

[132] V. W. M. van Hinsbergh, P. Koolwijk Endothelial sprouting and angiogenesis: matrix metalloproteinases in the lead. Cardiovasc. Res. 78(2) (2008), 203-212.

[133] R. F. M. Van Oers, E. G. Rens, D. J. LaValley, C. A. Reinhart-King, R. M. H. Merks Mechanical cell-matrix feedback explains pairwise and collective endothelial cell behavior in vitro. PLoS Comput. Biol. 10(8) (2014), e1003774. 
[134] H. Van Oosterwyck Computational mechanobiology: may the force be with you. J. Math. Biol. (2014), Epub ahead of print, DOI 10.1007/s00285-014-0795-6.

[135] B. Vanderlei, J. J. Feng, L. Edelstein-Keshet A computational model of cell polarization and motility coupling mechanics and biochemistry. Multiscale Model. Simul. 9(4) (2010), 1420-1443.

[136] M. Vicente-Manzanares, C. K. Choi, A. R. Horwitz Integrins in cell migration - the actin connection. J. Cell Sci. 122(Pt 2) (2009), 199-206.

[137] M. Vicente-Manzanares, X. Ma, R. S. Adelstein, A. R. Horwitz Non-muscle myosin II takes centre stage in cell adhesion and migration. Nat. Rev. Mol. Cell Biol. 10(11) (2009), 778790.

[138] M. Vicente-Manzanares, D. J. Webb, A. R. Horwitz Cell migration at a glance. J. Cell Sci. 118(Pt 21) (2005), 4917-4919.

[139] R. H. Wade, A. A. Hyman Microtubule structure and dynamics. Curr. Opin. Cell Biol. 9(1) (1997), 12-17.

[140] N. Wang, J. D. Tytell, D. E. Ingber Mechanotransduction at a distance: mechanically coupling the extracellular matrix with the nucleus. Nat. Rev. Mol. Cell Biol. 10(1) (2009), 75-82.

[141] E. S. Welf, J. M. Haugh Signaling pathways that control cell migration: models and analysis. Wiley Interdiscip. Rev. Syst. Biol. Mediciine 3(2) (2011), 231-240.

[142] E. S. Welf, H. E. Johnson, J. M. Haugh Bidirectional coupling between integrin-mediated signaling and actomyosin mechanics explains matrix-dependent intermittency of leadingedge motility. Mol. Biol. Cell 24(24) (2013), 3945-3955.

[143] B. Wojciak-Stothard, A. J. Ridley Rho GTPases and the regulation of endothelial permeability. Vascul. Pharmacol. 39(4-5) (2002), 187-199.

[144] C. W. Wolgemuth, J. Stajic, A. Mogilner Redundant mechanisms for stable cell locomotion revealed by minimal models. Biophys. J. 101(3) (2011), 545-553.

[145] C. W. Wolgemuth, M. Zajac The moving boundary node method: a level set-based, finite volume algorithm with applications to cell motility. J. Comput. Phys. 229(19) (2010), 72877308.

[146] L. Wood, R. Kamm, H. Asada Stochastic modeling and identification of emergent behaviors of an Endothelial Cell population in angiogenic pattern formation. Int. J. Rob. Res. 30(6) (2011), 659-677. 
[147] L. Yan, M. A. Moses, S. Huang, D. E. Ingber Adhesion-dependent control of matrix metalloproteinase-2 activation in human capillary endothelial cells. J. Cell Sci. 113(Pt 22) (2000), 3979-3987.

[148] A. L. Zajac, D. E. Discher Cell differentiation through tissue elasticity-coupled, myosindriven remodeling. Curr. Opin. Cell Biol. 20(6) (2008), 609-615.

[149] G. Zeng, S. M. Taylor, J. R. McColm, N. C. Kappas, J. B. Kearney, L. H. Williams, M. E. Hartnett, V. L. Bautch Orientation of endothelial cell division is regulated by VEGF signaling during blood vessel formation. Blood 109(4) (2007), 1345-1352.

[150] F. Ziebert, I. S. Aranson Effects of adhesion dynamics and substrate compliance on the shape and motility of crawling cells. PLoS One 8(5) (2013), e64511.

[151] F. Ziebert, S. Swaminathan, I. S. Aranson Model for self-polarization and motility of keratocyte fragments. J. R. Soc. Interface 9(70) (2012), 1084-1092. 